National Bureau of Standards

Iibrary, N.W. BIdg

DEC 71965

\title{
NBS
}

Eechnical Note

no. 325

\section{NUMERICAL SIMULATION OF IONOSPHERIC WAVE INTERACTION EXPERIMENTS}

THOMAS M. GEORGES

U. S. DEPARTMENT OF COMMERCE

NATIONAL BUREAU OF STANDARDS 


\section{THE NATIONAL BUREAU OF STANDARDS}

The National Bureau of Standards is a principal focal point in the Federal Government for assuring maximum application of the physical and engineering sciences to the advancement of technology in industry and commerce. Its responsibilities include development and maintenance of the national stand. ards of measurement, and the provisions of means for making measurements consistent with those standards; determination of physical constants and properties of materials; development of methods for testing materials, mechanisms, and structures, and making such tests as may be necessary, particularly for government agencies; cooperation in the establishment of standard practices for incorporation in codes and specifications; advisory service to government agencies on scientific and technical problems; invention and development of devices to serve special needs of the Government; assistance to industry, business, and consumers in the development and acceptance of commercial standards and simplified trade practice recommendations; administration of programs in cooperation with United States business groups and standards organizations for the development of international standards of practice; and maintenance of a clearinghouse for the collection and dissemination of scientific, technical, and engineering information. The scope of the Bureau's activities is suggested in the following listing of its four Institutes and their organizational units.

Institute for Basic Standards. Applied Mathematics. Electricity. Metrology. Mechanics. Heat. Atomic Physics. Physical Chemistry. Laboratory Astrophysics. * Radiation Physics. Radio Standards Laboratory:* Radio Standards Physics; Radio Standards Engineering. Office of Standard Reference Data.

Institute for Materials Research. Analytical Chemistry. Polymers. Metallurgy. Inorganic Materials. Reactor Radiations. Cryogenics. ${ }^{*}$ Materials Evaluation Laboratory. Office of Standard Reference Materials.

Institute for Applied Technology. Building Research. Information Technology. Performance Test Development. Electronic Instrumentation. Textile and Apparel Technology Center. Technical Analysis. Office of Weights and Measures. Office of Engineering Standards. Office of Invention and Innovation. Office of Technical Resources. Clearinghouse for Federal Scientific and Technical Information.* *

Central Radio Propagation Laboratory.* Ionospheric Telecommunications. Tropospheric Telecommunications. Space Environment Forecasting. Aeronomy.

* Located at Boulder, Colorado 80301.

* Located at 5285 Port Royal Road, Springfield, Virginia 22171. 


\title{
NATIONAL BUREAU OF STANDARDS \\ Eechnical Mote.325 \\ ISSUED October 25,1965
}

\section{NUMERICAL SIMULATION OF IONOSPHERIC WAVE INTERACTION EXPERIMENTS}

\author{
Thomas M. Georges \\ Institute for Telecommunication Sciences and Aeronomy \\ Environmental Science Services Administration \\ National Bureau of Standards \\ Boulder, Colorado
}

\begin{abstract}
NBS Technical Notes are designed to supplement the Bureau's regular publications program. They provide a means for making available scientific data that are of transient or limited interest. Technical Notes may be listed or referred to in the open literature.
\end{abstract}

For sale by the Superintendent of Documents, U.S. Government Printing Office Washington D.C. 20402

Price: $30 \notin$ 

The Fejer [1955] pulse interaction experiment is simulated numerically by solving, in an iterative fashion, the electron energy balance and magnetoionic propagation equations over a height-time "matrix" representing a disturbed ionosphere. The complex interdependence of physical variables is more accurately represented by including "selfdistortion" effects on the disturbing pulse, and allowing electron collision frequency and the energy loss coefficient $(G)$ to vary with electron energy and time. Provision is made for nonrectangular disturbing pulses as well as finite-length probing pulses. Phase as well as amplitude interaction effects are calculated.

Absorption and phase shift computations are based on the AppletonHartree or Sen-Wyller complete (arbitrary angle of propagation) formulations, but the insertion of more sophisticated developments in interaction theory, including electron density perturbations, should be possible without appreciable increase in program complexity.

Representative calculations demonstrate the effects of variations in experimental parameters, perturbations of ionospheric conditions, and various interpretational as sumptions.

The numerical approach offers advantages of simplicity and flexibility to those engaged in design and interpretation of wave interaction experiments. Synthesis of D-region electron density and collision frequency profiles, using the technique "in reverse," should prove superior to presently used trial-and-error methods.

Key Words: Cross modulation, D region, ionosphere, Luxembourg Effect, nonlinear propagation, wave interaction 



\section{Contents}

Abstract

1. INTRODUCTION

2. LIMITATIONS OF EXISTING ANALYSES

2. 1 The Experiment 2

2. 2 Analytical Interpretation 4

2.2.1 The $\Delta \nu$ Term 5

2.2.2 The $\partial \mathrm{k} / \partial \nu$ Term 7

2.2.3 Assumptions 8

3. NUMERICAL SIMULATION TECHNIQUES 9

4. SOME SAMPLE CALCULATIONS 19

4. I Variations in Disturbing Pulse Length 23

4. 2 Variations in Frequency and Polarization 24

4. 3 Dependence on Disturbing Frequency 24

4. 4 Neglecting Self-distortion and Energy $\begin{array}{ll}\text { Dependences of } G, \nu & 27\end{array}$

4. 5 Dependence on Form at G(Q) 27

4.6 Perturbations of the Electron Density Profile 30

4. 7 Disturbing Wave Self-distortion 30

4. 8 Use of the Generalized Theory 33

4.9 Gyro-interaction Effects 33

5. CONCLUSIONS AND LIMITATIONS 36

6. REFERENCES 40 


\section{Illustrations}

Page

1. Height-time representation of the Fejer experiment

2a. Operation of the simulation program

2b. Height-time-energy representation of a disturbed ionosphere

3. Functional diagram of the simulation program

4. Reference phase and amplitude interaction "profiles"

5. Electron density profiles

6. Collision frequency and time constant profiles

7. Effect of disturbing pulse length

8. Effect of frequency and polarization

9. Peak interaction vs. disturbing frequency (day)

10. Energy dependence of $G$

11. Interaction for various $G(Q)$

12. Interaction for bulges in $N(h)$ profile

13. Self-distortion of disturbing pulse

14. Interaction using generalized theory

15. Peak interaction vs. disturbing frequency (night)

16. Collision frequency perturbations for gyro-interaction 


\section{NUMERICAL SIMULATION OF IONOSPHERIC WAVE INTERACTION EXPERIMENTS}

Thomas M。Georges

\section{INTRODUCTION}

The present deficiency in the state of knowledge of the ionospheric D region has revived an interest in the cross modulation or radio wave interaction technique for the measurement of ionosphere electron density and collision frequency. Since the discovery of the Luxembourg Effect in 1933, numerous researchers have attempted to demonstrate the feasibility of utilizing cross modulation to obtain accurate ionospheric profiles [Fejer et al., 1955, 1959; Rumi el al., 1958, 1960, 1961, 1962a, 1962b; Barrington et al. , 1962a, 1962b, 1963; Titheridge, 1962; Smith et al., 1952, 1964]. However, the availability of very high-power radio pulse transmitters and sophisticated informationprocessing schemes has only recently indicated that sufficient experimental accuracy is obtainable. The necessity of using such high power to disturb the ionosphere leads to an examination of certain approximations implicit in present analytical methods for the interpretation of experimental results. It will be demonstrated that it is not feasible to account adequately for the complex interdependence of physical variables in a purely analytical fashion, and that numerical techniques provide a convenient and flexible means of interpreting ionospheric pulse interaction experiments. This work outlines a technique for the simulation of pulse interaction using a digital computer, and shows the dependence of measured quantities on experimental variables and some common interpretational assumptions. A forthcoming paper will demonstrate the application of this method to the synthesis of ionosphere profiles from interaction data. 


\section{LIMITATIONS OF EXISTING ANALYSES}

\subsection{The Experiment}

The experimental technique for obtaining ionosphere profiles using cross modulation is based on the familiar pulse interaction method developed by Fejer [1955]. A very high-power, ground-based "disturbing" transmitter sends a short pulse of electromagnetic energy vertically into the ionosphere, imparting momentary increases in average electron energy as the wave is partially absorbed at each height. This alteration in electron energy, through an increase in average collision frequency with air molecules, results in a perturbation of the wave absorption and phase coefficients of the medium. If a second pulse from a lower-power transmitter is caused to traverse the disturbed region in a downward direction (after reflection from a higher layer), in effect "meeting" the upward-traveling disturbing pulse, a perturbation of the received amplitude and phase of this probing pulse will occur. (The word "probing" is felt to be more appropriate to this experiment than "wanted," which originated in the terminology of the Luxembourg Effect.) This perturbation is characteristic of the disturbed conditions in a narrow height range near the meeting height. If this sequence of probing and disturbing pulses is repeated, with the probing pulses occurring twice as often as the disturbing ones, the relation between adjacent probing pulses will contain the perturbation information which can be interpreted in terms of electron density and collision frequency near the meeting height. It is seen from figure 1 , which illustrates the experiment, that the height of interaction can be adjusted by varying the relative delay time between pulses, and that height resolution depends principally on the length of the probing pulse. 


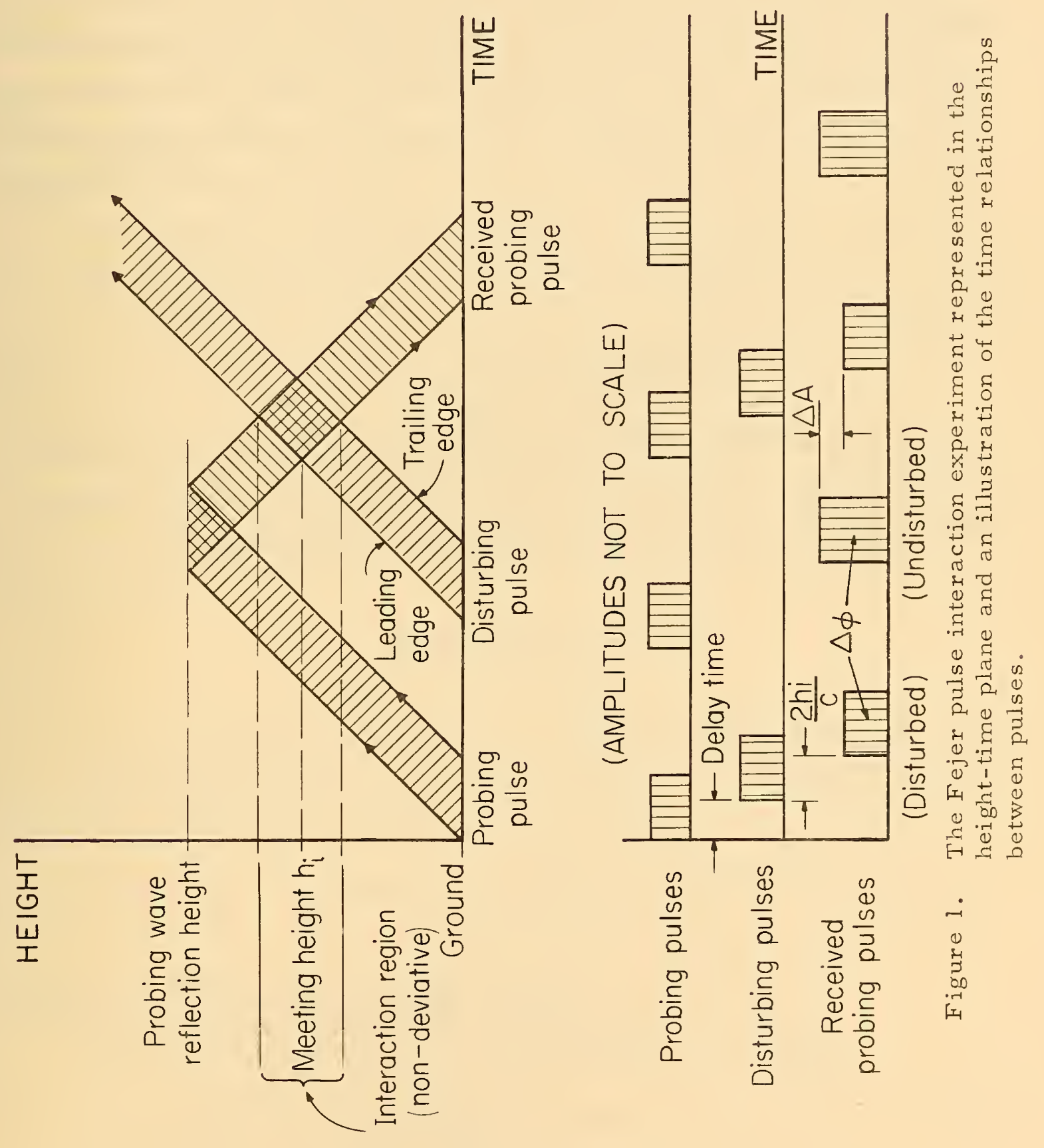


The study of the "nonlinear" behavior of the ionospheric plasma has recently expanded to consider possible enhancements of electron density due to perturbations of electron attachment coefficients and effects associated with compressibility of the medium such as the generation of several varieties of plasma waves, as well as the more familiar process involving alterations in effective electron collision frequency. However, only the last effect will be considered here, since the others are believed to be of secondary importance in the D region.

\subsection{Analytical Interpretation}

In the interpretation of this experiment, the pulse interaction process is usually represented by an integration of the perturbation in propagation constant over the height range of interaction:

$$
T\left(h_{i}\right)=-\int_{0}^{h_{i}} \frac{\partial k_{p}}{\partial \nu} \Delta \nu d h
$$

where $T$ is the complex interaction coefficient [Ferraro, Lee, and Weisbrod, 1963 ]

$$
T=r_{a}+j T_{\varphi}
$$

$k_{p}$ is the complex propagation constant for the probing wave, $k_{p}=\alpha_{p}+j \beta_{p}$

$\nu \quad$ is the electron collision frequency with neutral molecules,

$h_{i}$ is called the interaction (or "meeting") height of the two pulses.

In terms of experimentally measured quantities,

$$
\mathrm{T}_{\mathrm{a}}=\frac{\Delta \mathrm{A}}{\mathrm{A}}
$$

often called the "modulation depth," and 


$$
\mathrm{T}_{\varphi}=\Delta \varphi \text { (radians) }
$$

where $\Delta \mathrm{A}$ and $\Delta \varphi$ are the received probing pulse amplitude and carrier phase perturbations as shown in figure $1 . T_{\varphi}$ has been defined with a plus sign in (1) in order to yield positive $T_{\varphi}$ for a $\Delta \varphi$ corresponding to phase advance. Thus $T_{\varphi}$ is negative for frequencies above the gyrofrequency.

\subsubsection{The $\Delta v$ Term}

The collision frequency perturbation, $\Delta \nu$, depends on the intensity of the electromagnetic field through the electron energy-gain process. For the relation between the energy of electrons and their collision frequency with air molecules, use is made of laboratory determinations of collision cross sections for air in the energy range of interest. Experiments of Phelps and Pack [1959] have shown that, to a good approximation,

$$
\Delta v=v\left(\frac{Q-Q_{0}}{Q}\right)
$$

The time dependence of $\nu$ can be obtained by solving the differential equation for electron energy balance,

$$
\frac{d Q}{d t}=\nu\left[\epsilon-G\left(Q-Q_{0}\right)\right]
$$

where $Q$ is the electron energy $=a k \theta$,

Qo is the electron equilibrium (undisturbed) energy,

$k$ is Boltzmann's constant,

$\theta$ is electron temperature,

$\varepsilon \quad$ is the energy gained from the wave by each electron per mean free time, 
a is a constant relating temperature to the energy distribution

$G$ is the electron energy loss coefficient, the fraction of excess energy lost per collision (usually determined by laboratory measurements).

All of the variables in this equation are really probability distributions, of which some statistical moment is usually used for computations. Current practice is to use the most probable values, in which case $a=1$, and $\nu$ is called $\nu_{\mathrm{m}}$.

The general solution of (4) is, for $G$ and $\nu$ nearly constant within the interval $Q_{0}$ to $Q$,

$$
Q(t)=\frac{\epsilon}{G}[1-\exp (-t / T)]+Q_{0}
$$

for conditions of energy gain, that is, when the medium is absorbing energy from the disturbing pulse, and

$$
Q(t)=\frac{\epsilon\left(\tau_{d}\right)}{G} \exp \left(\frac{T_{d}-t}{T}\right)+Q_{0}
$$

during the decay period after the pulse has passed. Here $T=1 / G \nu$ and $\tau_{d}$ is the disturbing pulse length. Equations (5) are expressed in terms of the height $h$ of the probing pulse leading edge by substituting

$$
t=2 \frac{h_{i}-h}{c}
$$

where $h_{i}$ is the meeting height of leading edges, C is the free-space propagation velocity.

In (4) and (5), G is usually treated as a constant equal to about $10^{-3}$, although various forms for the energy dependence of the loss 
term have been considered in order to account for inelastic collisions [Huxley, 1953; Altshuler, 1963; Benson, 1964]. The energy gain ter $\mathrm{m}, \epsilon$, is obtained by calculating the disturbing wave energy absorbed at each height, per electron, per mean free time:

$$
\varepsilon(h)=\frac{P_{d}^{\alpha} d}{N v}
$$

where $p_{d}$ is the disturbing wave power density at $h$,

$$
P_{d}(h)=\frac{P_{d}}{4 \pi h^{2}} \cdot \exp \left(-\int_{0}^{h} \alpha_{d} d h^{\prime}\right)
$$

$\mathrm{N}$ is the electron density,

$\alpha_{d}$ is the disturbing wave power absorption coefficient at $h$,

$\mathrm{P}_{\mathrm{d}}$ is the effective vertically radiated disturbing power.

$$
\text { 2.2.2 The } \partial \mathrm{k}_{\mathrm{p}} / \partial \nu \text { term }
$$

The term $\partial \mathrm{k}_{\mathrm{p}} / \partial \nu$ in (1) is obtained from some suitable expression for the complex refractive index of a uniform ionized medium in a magnetostatic field. At this time, only the so-called Quasi-Longitudinal approximations to the Appleton-Hartree and the Sen and Wyller [1960] generalized theories have been so treated [Benson, 1963, 1964]. When the former theory is used, for example, an expression for the real part of $\partial k_{p} / \partial \nu$ is of the relatively simple form

$$
\frac{\partial \alpha_{p}}{\partial \nu}=A N \cdot \frac{\left(\omega \pm \omega_{L}\right)^{2}-\nu^{2}}{\left[\left(\omega \pm \omega_{L}\right)^{2}+\nu^{2}\right]^{2}}
$$

where $\omega$ is the probing wave angular frequency,

$\alpha_{p}$ is the probing wave power absorption coefficient, 
$\omega_{L}$ is $2 \pi f_{h} \cos Y$,

$f_{h}$ is the electron cyclotron frequency,

$Y \quad$ is the angle of propagation with respect to the geomagnetic field

$\mathrm{A}=1.062 \times 10^{-5}$ in the m.k.s. system of units

$\mathrm{N}$ is the electron density

$\nu \quad$ is the effective collision frequency.

It is known, however, that such simplifications of the magnetoionic theory are not strictly applicable under some conditions normally encountered in the ionosphere. Moreover, recent developments in the theory of nonlinear wave propagation indicate that new expressions based on a non-Maxwellian electron velocity distribution may be required for waves of intensities which will be considered here [Layzer and Menzel, 1965, and Budden, 1965]. For these theories, it may not be possible to obtain expressions for $\partial \mathrm{k}_{\mathrm{p}} / \partial \nu$ directly.

\subsubsection{Assumptions}

It should now be evident that with a proper substitution of (9), $(8),(7),(6),(5 a),(5 b)$, and (3) into (1) the integration (even numerically) could become quite involved due to the complicated interdependence of variables. In practice, at least one of the following rather limiting assumptions is usually made:

1. The disturbing pulse does not undergo "self-interaction"; that is, no dependence of $\alpha_{d}$ in (8) on time or electron energy is allowed.

2. Electron energy rise and decay follow a simple exponential behavior in time; that is, energy and time dependences of $G$ and $\nu$ are neglected in computing $\epsilon / G$ and $T=1 / G \nu$ in (5).

3. The energy loss coefficient $G$ is constant. 
4. The disturbing pulse is rectangular, a condition seldom encountered in practice.

5. Probing pulses are infinitesimally short.

6. The difficulty of evaluating $\partial \mathrm{k}_{\mathrm{p}} / \partial \nu$ for more complete expressions for the refractive index restricts application to the limitations of the approximate formulas.

Although the first three of these approximations are probably not severe limitations under conditions of even moderately high disturbing power, it is of interest to know within what disturbing frequency and power ranges these approximations remain justified. Although the applicability of 4,5 , and 6 clearly depends on the individual experiment, it appears that in general it would be desirable to remove these restrictions.

\section{NUMERICAL SIMULATION TECHNIQUES}

The nonlinearity of the analytical expressions which describe the wave interaction process, and the complex interdependence of the physical quantities involved, suggest a numerical approach to the solution of practical problems. For this purpose a digital computer program has been developed which simulates the upward passage of a single disturbing pulse through a model ionosphere, its effect on the medium, and the effect of the medium on a single downcoming probing pulse. This approach has the advantages that all the approximations mentioned above are unnecessary, and that a great deal of flexibility is posible in the selection of experimental parameters and method of treatment.

It will be assumed that some suitable expression for the complex refractive index of a uniform magnetoionic medium has been chosen, and that it is desired to apply it to a medium which can undergo only 
small íme variations in a wave period, and where only small space gradients exist with respect to a wavelength. Under such conditions, generally satisfied at high frequencies, the principles of ray theory can usually be applied, wherein the medium is considered equivalent to a large number of space and time "cells" within which the macroscopic properties of a uniform medium can be used. Overall nonlinear effects can then be simulated by allowing the conditions in each cell to depend on conditions in previous cells and on the disturbing wave power density.

Since the Fejer interaction experiment involves essentially vertical propagation, only two dimensions need be considered -- height and time. The disturbed medium can then be completely represented as a two-dimensional height-time "matrix," with each cell containing some characteristic of the disturbed medium, such as electron temperature. For ray theory to be accurate it is necessary that space and time variations of the medium be small within each cell. Ionosphere parameters do not change appreciably within height and time intervals of 1 kilometer by $31 / 3$ microseconds (the time for a wave to travel 1 kilometer in free space), and this convention has been adopted for convenience. It will be as sumed that disturbing pulses are so much shorter than effective recombination times that electron and neutral particle density as well as gas temperature do not vary with time, and these profiles, presently defined between 20 and 120 kilometers, are entered into the program in tabular form in 1 kilometer steps. Electron collision frequency is calculated from the electron temperature (energy) and neutral particle density, rather than by a direct insertion of a $\nu(h)$ profile. The expression for the complex refractive index is entered as a subprogram which can be varied independently, as are the dependences of $G(Q)$ and $\nu(Q)$ which may be entered in functional or tabular form. 
Experimental control variables are: disturbing and probing frequency and polarization, disturbing power and pulse length, local gyrofrequency and magnetic dip angle. The probing wave is represented by a series of infinitesimal "delta" pulses meeting the disturbing pulse leading edge at every 1 kilometer interval over the height range of interest. It is assumed that interaction occurs in a nearly nondeviative region; that is, the pulse group velocities effectively equal the free-space propagation velocity, c.

It is also assumed that electron energy distributions remain so nearly Maxwellian that the idea of an effective collision frequency as expressed in the Appleton or Sen-Wyller [1961] developments is applicable.

The operation of the program is most easily understood by reference to figures $2 \mathrm{a}$ and $2 \mathrm{~b}$, which illustrate the wave interaction region in the height-time plane, and figure 3 , an operational diagram of the program. Assuming only a free-space divergence of power between the ground and $h_{1}$, the disturbing power density, $\mathrm{p}_{\mathrm{d}}$, is calculated for the first (lowest) height interval, $h_{1}$, and the pulse waveform is digitally represented by a one-dimensional array containing values of $\mathrm{p}_{\mathrm{d}}$ for each time cell. Any pulse shape can be defined at this point, and the number of time cells required depends on the pulse length. This waveform and the atmospheric parameters for the first height interval are then used to calculate the time-dependent electron energy for each time interval, using a form of (5) suitable for numerical computations,

$$
Q(t+\Delta t)=Q(t)+\left[Q_{0}+\frac{\epsilon(t)}{G(t)}-Q(t)\right]\left[1-\exp \left(\frac{-\Delta t}{T(t)}\right)\right]
$$




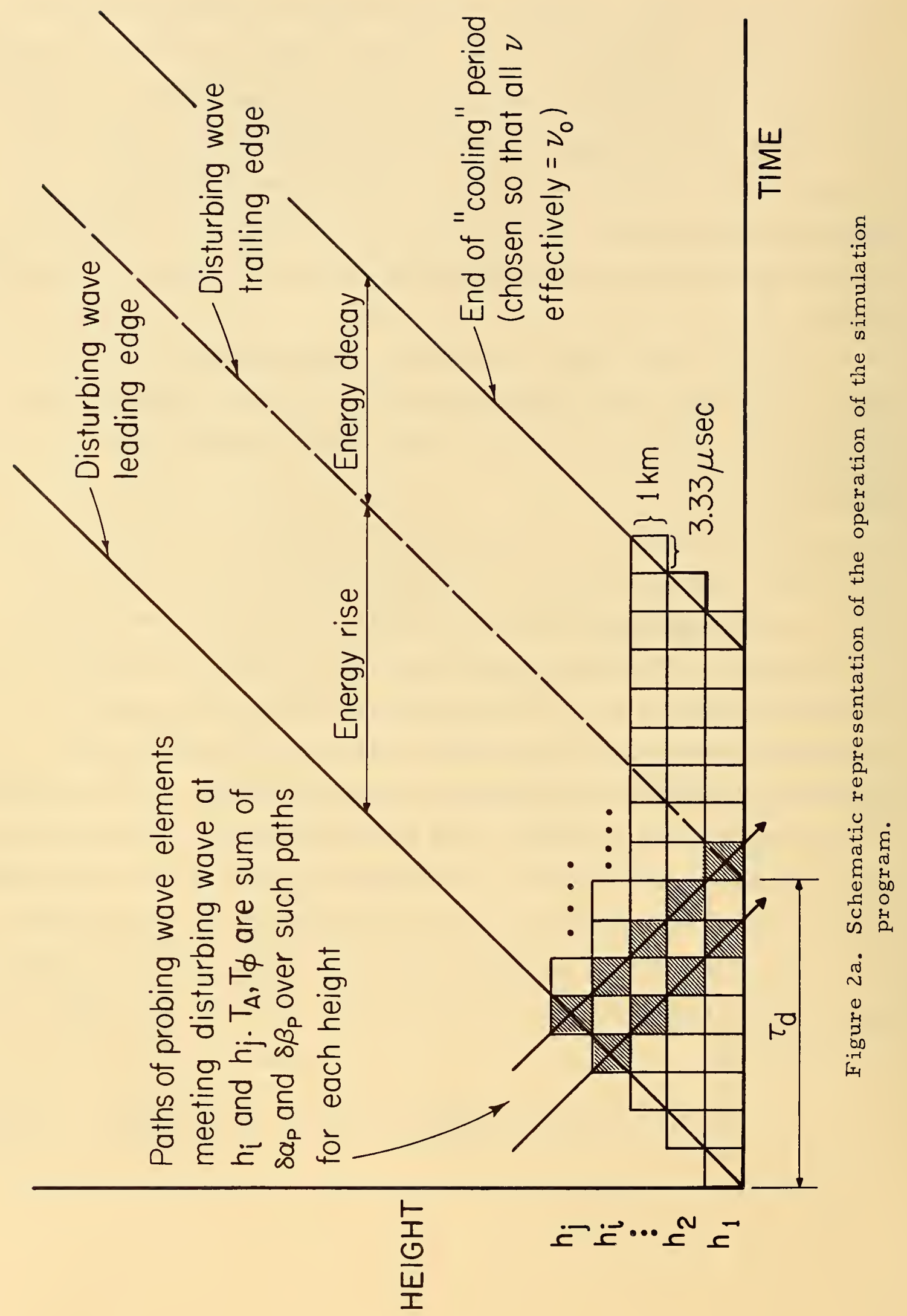




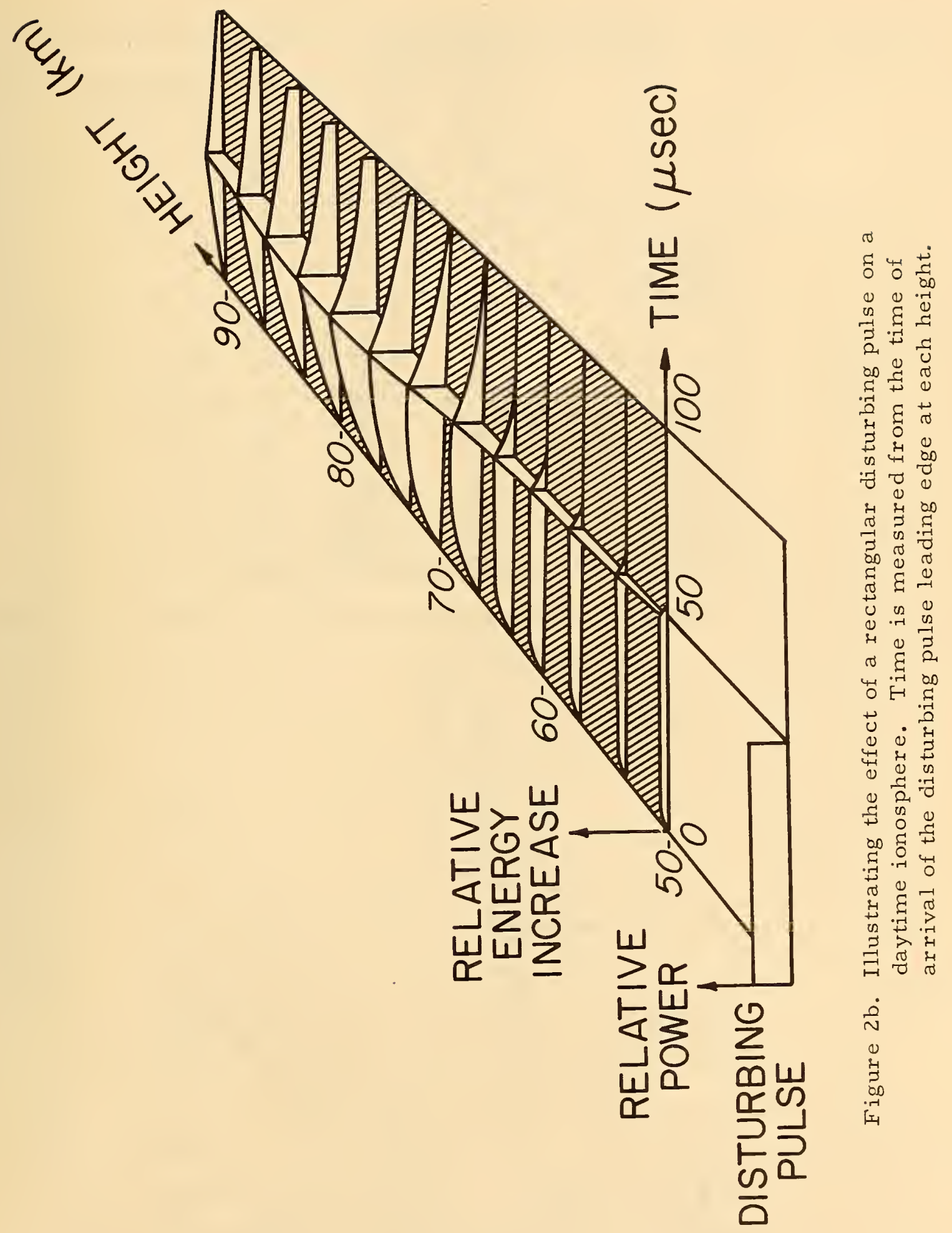




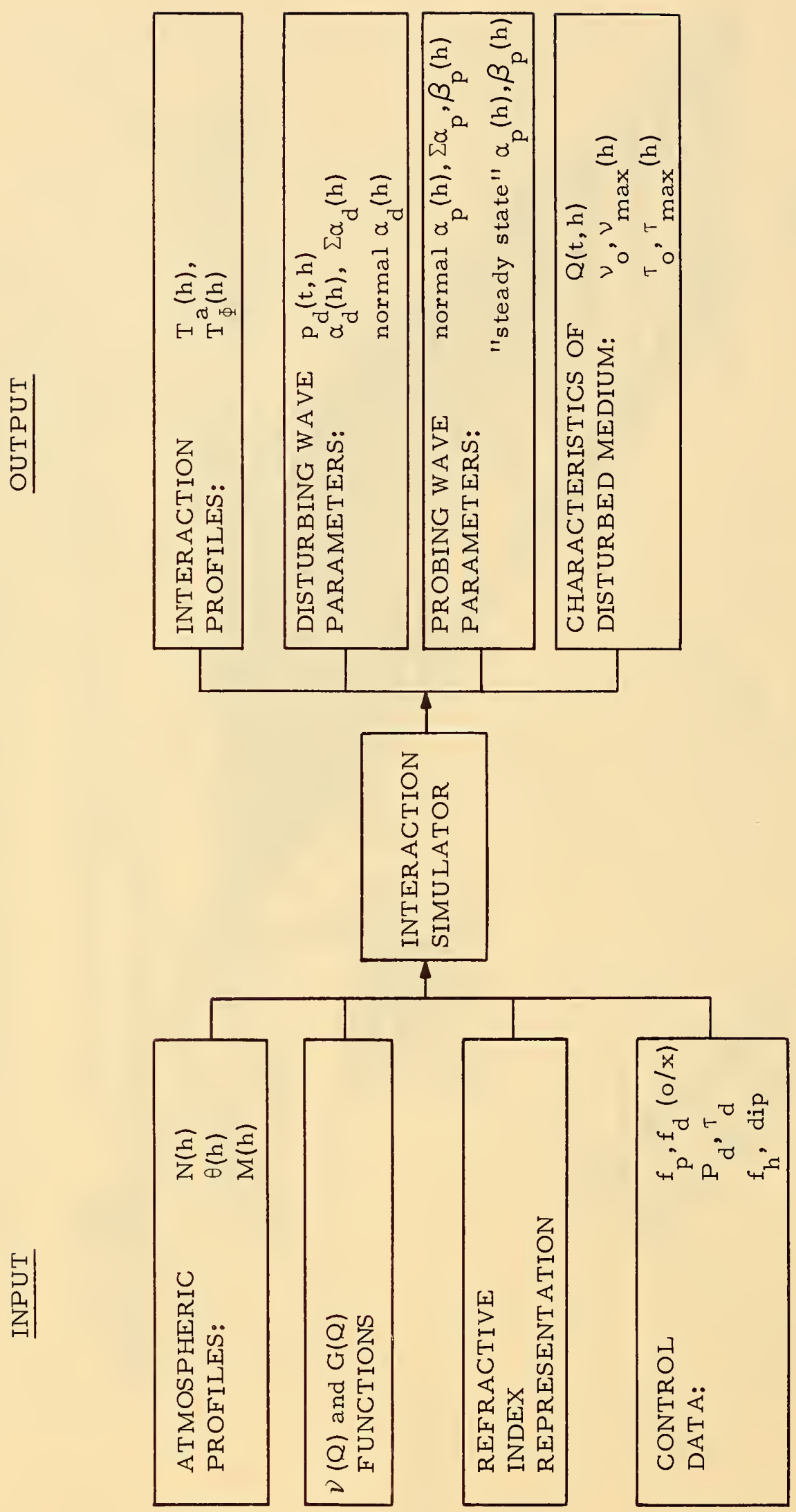

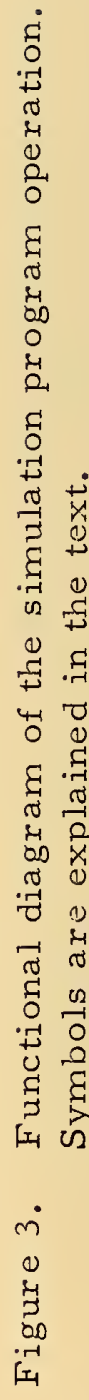


where $\Delta t$ is a time interval sufficiently small that $Q, G(Q)$ and $\nu(Q)$ do not change appreciably within it,

$$
\begin{aligned}
& T(t)=\frac{1}{G(t) \nu(t)} \\
& \epsilon(t)=6.242 \times 10^{18} \cdot \frac{p_{d}^{\alpha} d}{N \nu} \text { for } 0 \leq t \leq \tau_{d} \text {; zero otherwise, } \\
& \epsilon \quad \text { is in electron volts, } \\
& p_{d} \text { is in watts } / \mathrm{cm}^{2}, \\
& N \quad \text { is in } \mathrm{cm}^{-3} \\
& \alpha_{d} \text { is in nepers/cm, } \\
& \nu \quad \text { is in sec } \\
& Q_{0} \quad=\text { ak } \theta_{0}(h) .
\end{aligned}
$$

Using this expression in an iterative fashion, each time cell at $h_{1}$ can be successively filled with a value for electron energy, from which new values of $G$ and $\nu$ are calculated for the next time cell via the $G(Q)$ and $\nu(Q)$ functions. In this way, the energy dependences of these quantities are retained. The absorption coefficient, $\alpha_{d}$, for the disturbing wave and the absorption and phase coefficients, $\alpha_{p}$ and $\beta_{p}$, for the probing wave can now be calculated for each time cell at $h_{1}$ by using proper values of $\mathrm{N}$ and $\nu$ in the refractive index subprogram. When enough time cells are filled after $t=\tau_{d}$ that the electron energy has nearly cooled to undisturbed conditions again, $\alpha_{d}$ is applied to each element of the disturbing wave power density, so that when the 1 kilometer spherical divergence attenuation is included, the disturbing waveform is replaced by a new wavefor $m$ for the next height interval, $h_{2}$. This waveform is of course slightly distorted from its original shape because of the variations in $\alpha_{d}$ for each $h_{1}$ time cell. At $h_{2}$ the new power density waveform is applied to conditions at that height to fill its time cells as before with information on the propagation characteristics of the medium. 
This process is repeated throughout the region of interest, so that the resulting matrix covers the entire disturbed medium in height and time.

Knowing $k_{p}$ in each height-time cell, the interaction contribution of each cell can be calculated by subtracting the normal (undisturbed) value of $k_{p}\left(k_{p_{0}}\right)$ from the disturbed value:

$$
\delta k_{p}=k_{p}-k_{p_{0}}
$$

The total interaction suffered by each element of the probing wave is obtained by summing $\delta \mathrm{k}_{\mathrm{p}}$ over the diagonal set of matrix elements corresponding to each meeting height. The absorption encountered outside the interaction region is the same for both a disturbed and an undisturbed pulse, and is not included in the calculations since it does not affect the interaction coefficient. To obtain the interaction information, it is not necessary to store the entire two-dimensional matrix during computation, since it is known "in advance" which meeting height corresponds to each time cell at each height. The values for $\delta \alpha_{p}$ and $\delta \beta_{p}$ may thus be stored in appropriate locations corres ponding to each meeting height, immediately upon computation, and summation carried out automatically when the next value for that meeting height is encountered.

In the calculation of $\delta \alpha_{p}$ and $\delta \beta_{p}$ at each height it is necessary to perform calculations for the complex refractive index for the value of $\nu$ in each time-height cell, and it is often necessary to subtract values which differ by extremely small amounts. For larger differences it is possible to calculate values of, say, $k_{p}(\nu)$ and $k_{p}\left(\nu_{0}\right)$ and subtract to obtain their difference; however, when differences become small, as for very small interaction, this method is not satisfactory because of the limitations of accuracy inherent in digital computers. 
To calculate very small differences in refractive index, a numerical differentiation of $\mathrm{k}_{\mathrm{p}}$ with respect to $\nu$ is carried out over a sufficiently small interval of $\Delta \nu$ that

$$
\frac{\partial \mathrm{k} p}{\partial \nu} \approx \frac{\Delta \mathrm{k} p}{\Delta \nu}
$$

then

$$
\delta k_{p}=\frac{\Delta k}{\Delta \nu} \delta \nu
$$

where $\delta \nu$ is the actual difference in $\nu$ for which the value of $\delta k_{p}$ is desired. $\Delta \nu$ is taken to be sufficiently large that an accurate value of $\Delta \mathrm{k}_{\mathrm{p}} / \Delta \nu$ can be calculated, but still small enough that (ll) is satisfied. The principal output of the program is a tabulation of the phase and amplitude interaction coefficients, $\mathrm{T}_{\mathrm{a}}$ and $\mathrm{T}_{\varphi}$ for each meeting height where significant cross modulation occurs. These interaction coefficients (figure 4) may he interpreted either as perturbations to many very short probing pulses meeting the disturbing leading edge at each height, or as representing the distorted shape of a continuous probing wave received at the ground, such as in a cosmic noise or "sky temperature" cross modulation experiment.

Among the "by-products" of the interaction computations are the time functions of electron energy and disturbing power density waveform, collision frequency, heating time constant and probing and disturbing wave absorption parameters at each height, the approximate steady state conditions which would result from a very long disturbing pulse, and the total disturbed and undisturbed absorption for each wave. These provide additional insight into the physical processes involved. 

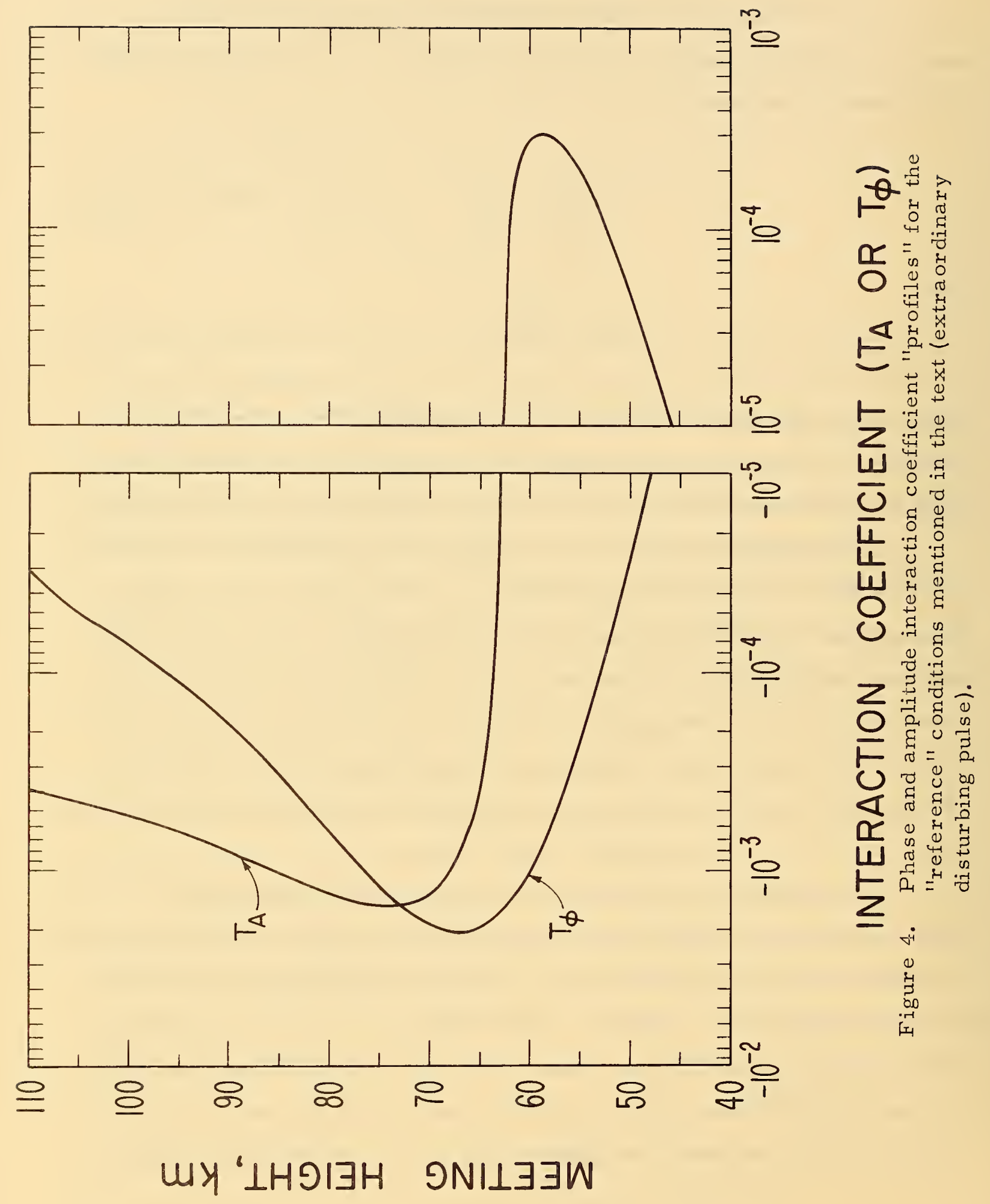


\section{SOME SAMPLE CALCULATIONS}

The numerical technique just described has been used to perform a series of sample calculations in order to demonstrate the effects of variations in experimental parameters and interpretational assumptions as well as to explore the general utility of the wave interaction experiment as a D-region diagnostic tool. In each of the results to be presented, a comparison is made between the amplitude interaction coefficient profile calculated from a set of "reference" conditions, and those calculated under the conditions or as sumptions in question. Phase interaction effects are shown only for the reference conditions, since they behave basically the same as the amplitude effects. The Appleton-Hartree expressions for the refractive index are used for most of these computations because of their simplicity; however, no essential differences in the points to be illustrated results from the use of more "complete" expressions. The reference conditions, chosen somewhat arbitrarily, but approximating conditions believed to be experimentally optimum, are as follows:

disturbing vertically radiated power $10^{7}$ watts disturbing frequency $10 \mathrm{Mc} / \mathrm{s}$ (extraordinary) disturbing pulse length 57 microseconds probing wave frequency $3 \mathrm{Mc} / \mathrm{s}$ (ordinary)

The reference electron density profile (figure 5, curve C) is a "mean midday" D-region profile for mid-latitudes resembling that in Knecht and Van Zandt [1964]. Neutral air density, $M(h)$, and gas temperature, $\theta(h)$, profiles are from the ARDC Standard Atmosphere (1959). The energy dependences of $G$ and $\nu$ are essentially the same as those of Megill [1965], and represent the results of drift tube experiments of Phelps cited therein. For small energy excursions these functions may 


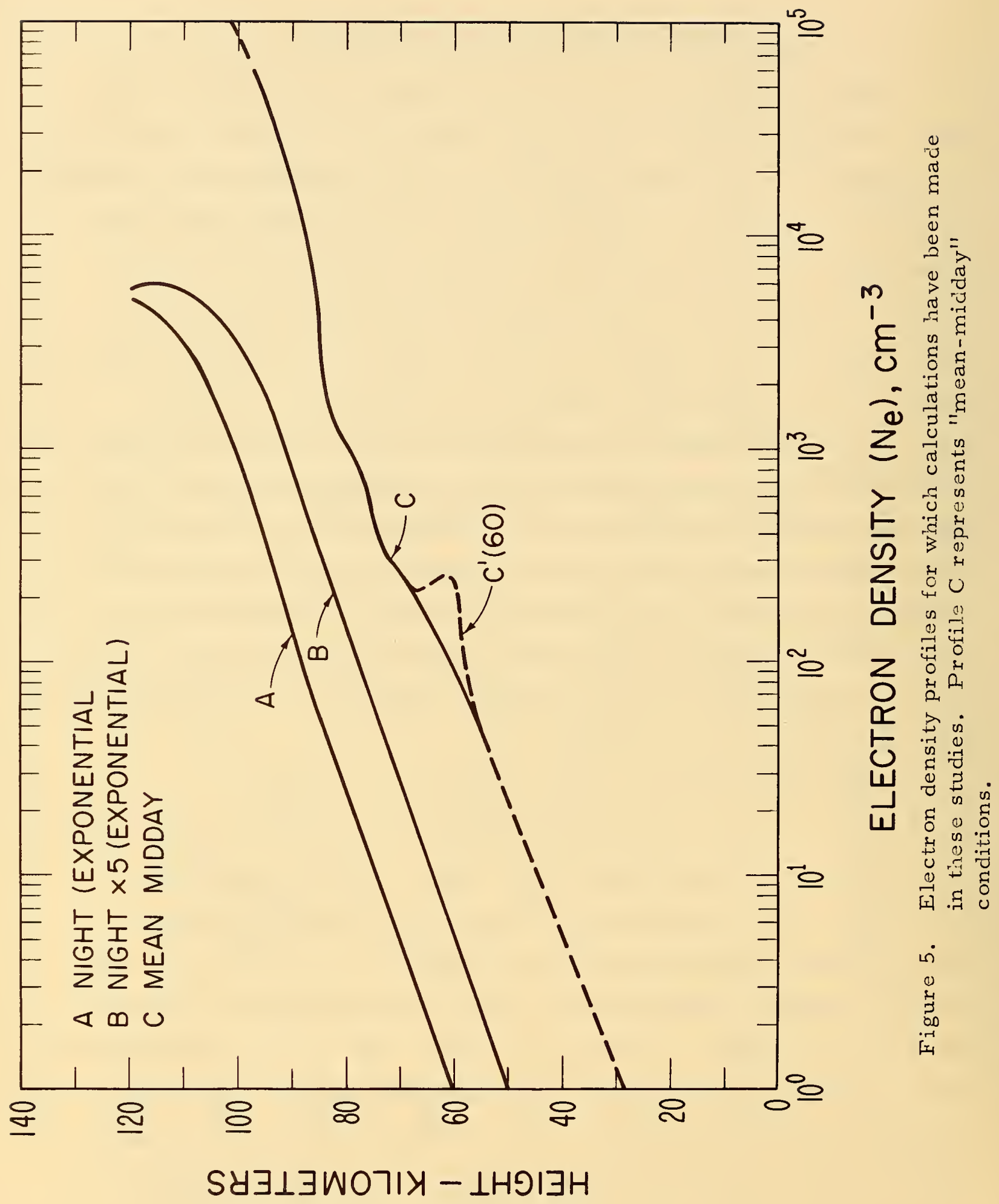




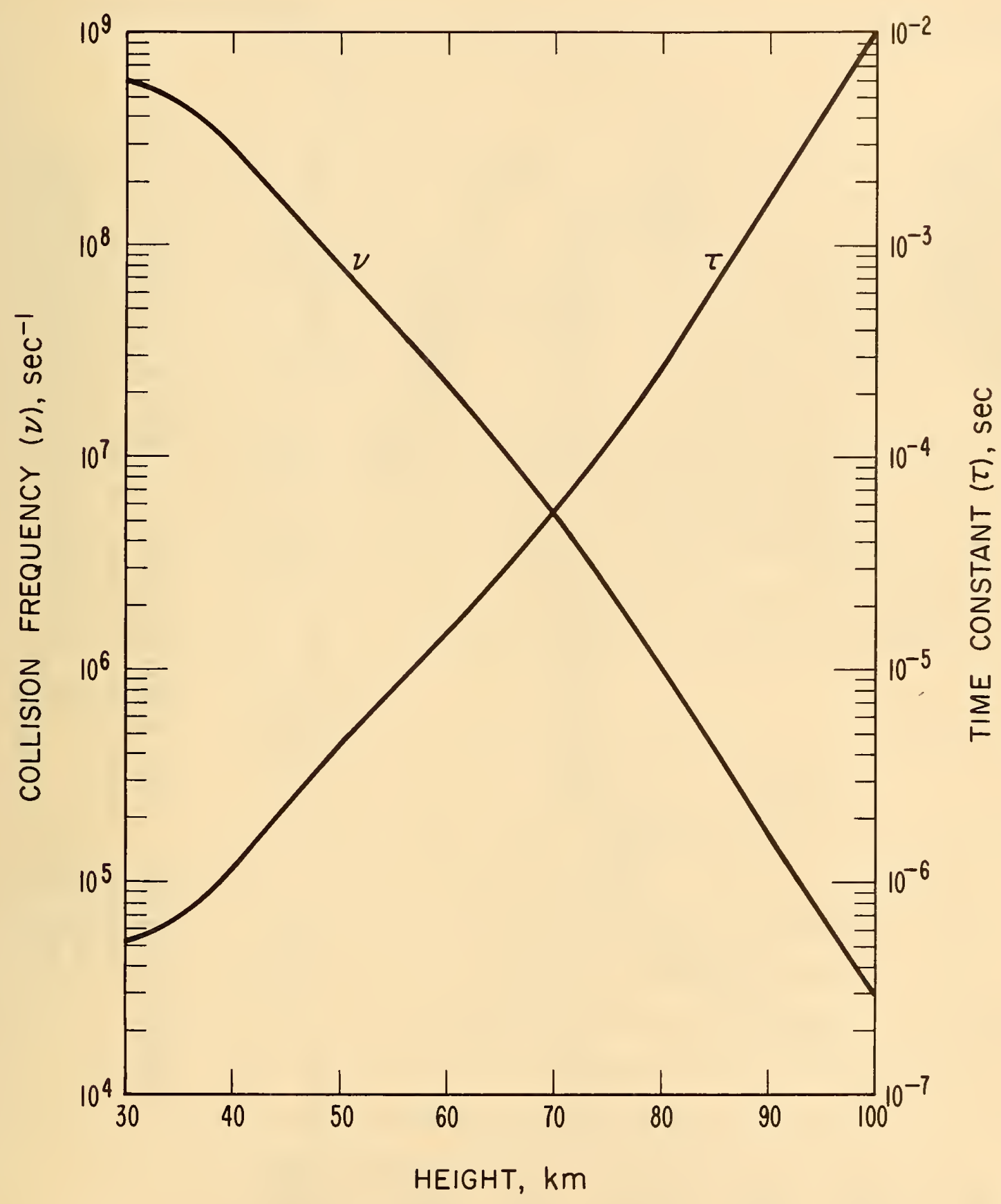

Figure 6. Profiles of electron collision frequency anc' heating time constant resulting from the atmospheric parameters assumed in these studies. 
be approximated by

$$
\begin{aligned}
& \nu(Q) \approx 9.7 \times 10^{-8} \mathrm{Q}^{.978} \mathrm{M} \\
& \mathrm{G}(\mathrm{Q}) \approx 4.7 \times 10^{-4} \mathrm{Q}^{-.55}
\end{aligned}
$$

It should be emphasized that present results for $G$ in particular are subject to considerable doubt, due to the difficulty of making laboratory measurements of the loss coefficient at such low electron energies; however, these will be taken as the best available. Recent cross modulation measurements [Smith, 1964, and Bourne, private communication] lend support to an approximately $Q^{-\frac{1}{2}}$ dependence of $G$ in the ionospheric electron energy range (.02 to .06 e.v.). Various other forms for the loss term in (4) have been proposed [Huxley, 1953; Altshuler, 1963], but it appears that these can be accounted for by just letting $G=G(Q)$ as is done here. Figure 6 shows the collision frequency and heating time constant profiles resulting from these atmospheric parameters.

\section{1 Variations in Disturbing Pulse Length}

Figure 7 shows the results of variations in the disturbing pulse length. The rather unconventional scale used for $\mathrm{T}_{\mathrm{a}}$ in this and following figures is a convenient way of illustrating a quantity which exhibitsvariations over a large range of positive and negative values, although it may unduly accentuate small interaction effects. As would be expected, longer pulses are more effective in causing heating in the upper $\mathrm{D}$ region because of the longer heating time constants there; and the height of zero interaction coefficient (integrated interaction) is increased because of increased positive interaction at lower heights. The shortest pulse length, on the other hand, provides little relative emphasis on lower heights, its principal effect apparently being only a reduction in overall sensitivity. The relation between the "crossover" 
heights of $\mathrm{T}_{\mathrm{a}}$ and the height of actual zero interaction demonstrates the difficulty of applying any simple relation to the crossover height to obtain the height of zero interaction as an independent determination of collision frequency there. It will be seen that this difference depends on other experimental parameters as well. The height of zero interaction, however, does not significantly depend on disturbing pulse length, since the heating time constant at this height is usually much shorter than pulse lengths ordinarily used, and a quasi-steady state electron energy is achieved quite rapidly.

\section{2 Variations in Frequency and Polarization}

Figure 8 shows the magnitude of variations to be expected as a result of changing probing and disturbing wave frequency and polarization as well as the electron density profile to one representing typical mid-latitude nighttime conditions. Of particular interest is the dependence on the disturbing frequency and polarization.

\section{3 Dependence on Disturbing Frequency}

Figure 9 shows the values of the maxima of the interaction coefficients plotted against disturbing frequency. Both amplitude and phase coefficients are shown for $\circ$ and $x$ disturbing polarizations. The extension of these computations to below the gyrofrequency serves more to illustrate the properties of the interaction expressions than to represent actual interaction experiments; the application of a ray treatment tends to lose its meaning as low frequencies are approached, due to partial reflection conditions and other strictly wave phenomena. Deviative effects at lower frequencies also tend to violate certain assumptions usually made in wave interaction experiments. 


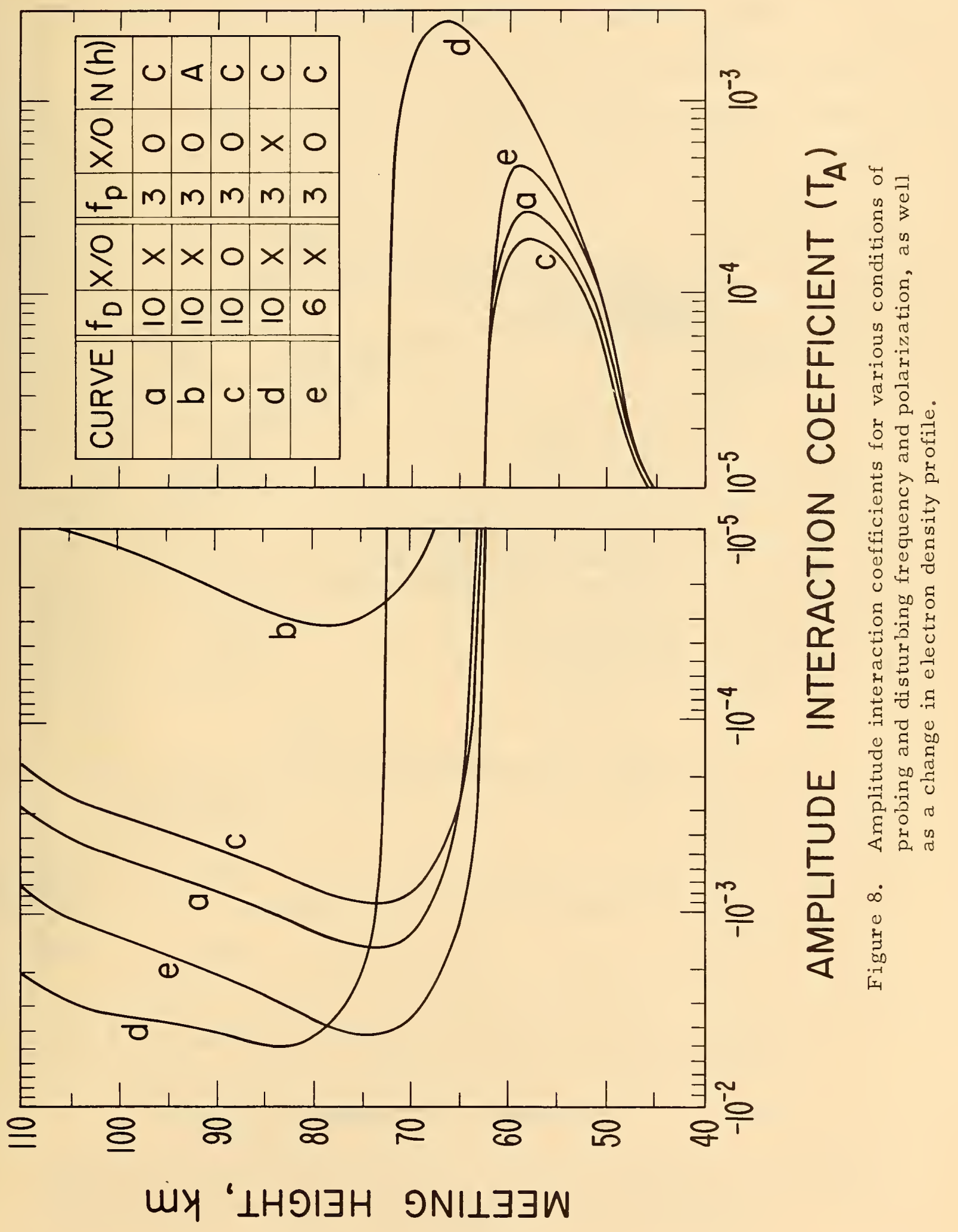




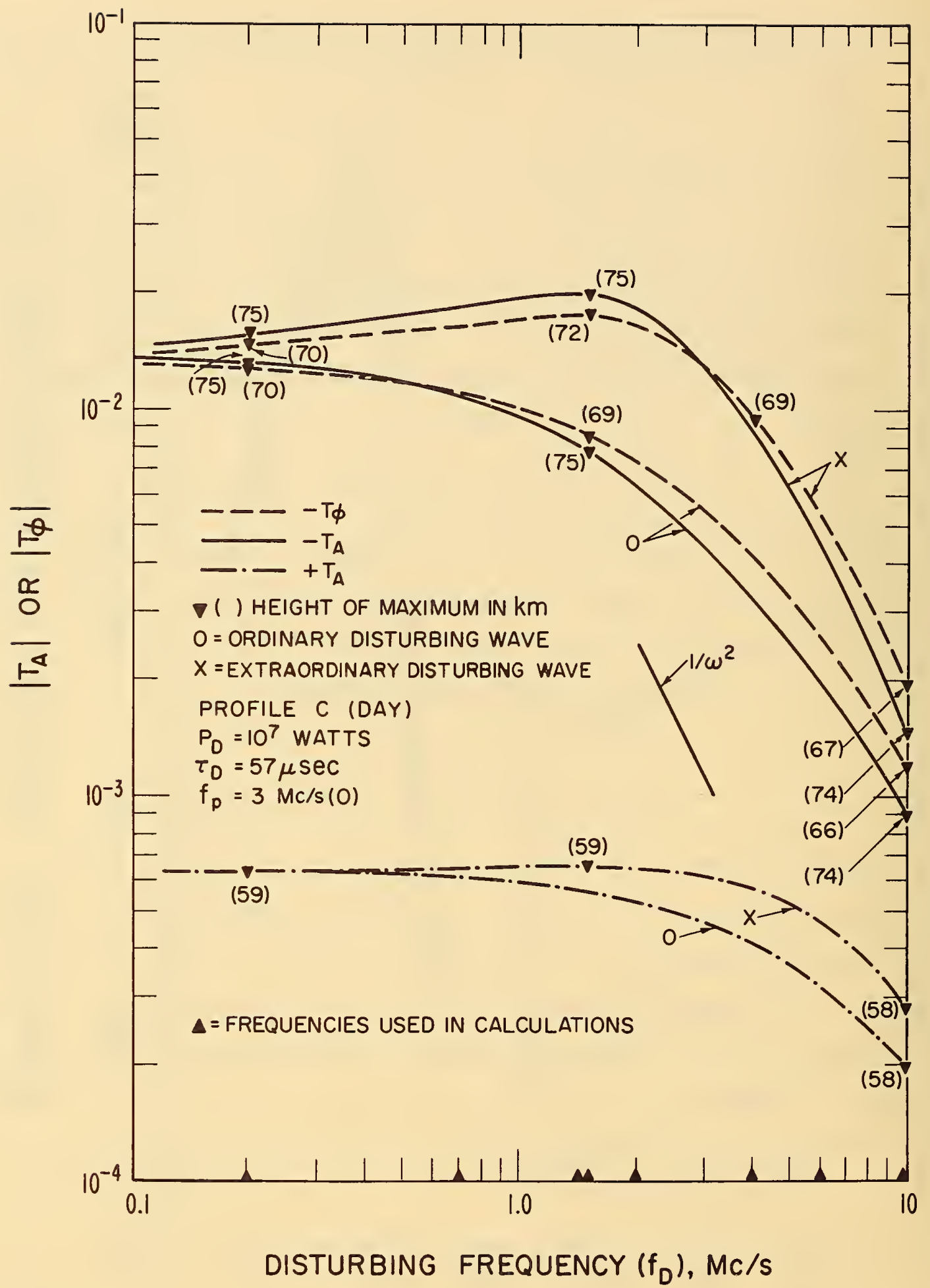

Figure 9. The maximum values of $\mathrm{T}_{a}\left(+\right.$ and - ) and $\mathrm{T}_{\varphi}$ for both disturbing polarizations as a function of disturbing frequency, showing the heights at which the maxima occur. These calculations were made using the Appleton-Hartree forumulas. 
4. 4 Neglecting Self-distortion and Inergy Dependences of $G, v$

Several calculations have been made in an attempt to show the errors which might result from neglecting the disturbing wave selfdistortion and nonlinear absorption, and in considering $G$ and $y$ to be constant at each height in (10). It is difficult to show in a concise manner the effects of these approximations in terms of all experimental parameters such as frequencies, power, and atmospheric profiles. In general, it appears that these effects do not become important until peak electron energy perturbations exceed about 100\%. Under the reference conditions (where energy perturbations do not exceed a few percent), neglect of disturbing wave self-distortion results in only about $+0.1 \%$ maximum error in interaction coefficient and the assumption of constant $G$ and $\nu$ at each height results in about -.85\% error. However, increasing the effective disturbing power from $10^{7}$ to $10^{9}$ watts increases these errors to $+6.6 \%$ and $-20 \%$, while decreasing the dis turbing frequency from $10 \mathrm{Mc} / \mathrm{s}$ to $3 \mathrm{Mc} / \mathrm{s}$ yields $-2.4 \%$ and $+2 \%$.

\section{5 Dependence on $F$ orm at $G(Q)$}

Several representations for the energy dependence of the electron energy loss coefficient, $G(Q)$, have been used to calculate interaction profiles for the purpose of determining the sensitivity of interaction to changes in $G$. The $G(Q)$ functions used in these calculations are illustrated in figure 10, and are rather arbitrary perturbations about several dependences which have been proposed. Results are expressed in terms of the amplitude interaction profiles in figure 11. The curve labeled $G_{1}(Q)$ is taken from Megill [1965]. The curve labeled $4.7 \times 10^{-4} \mathrm{Q}^{-.55}$ is an asymptotic approximation to $G_{1}(Q)$ and appears to give nearly identical interaction results when applied within the ionospheric electron energy range. Two other forms of $G$ show effects on 


\section{ELECTRON TEMPERATURE $(\theta),{ }^{\circ} \mathrm{K}$}

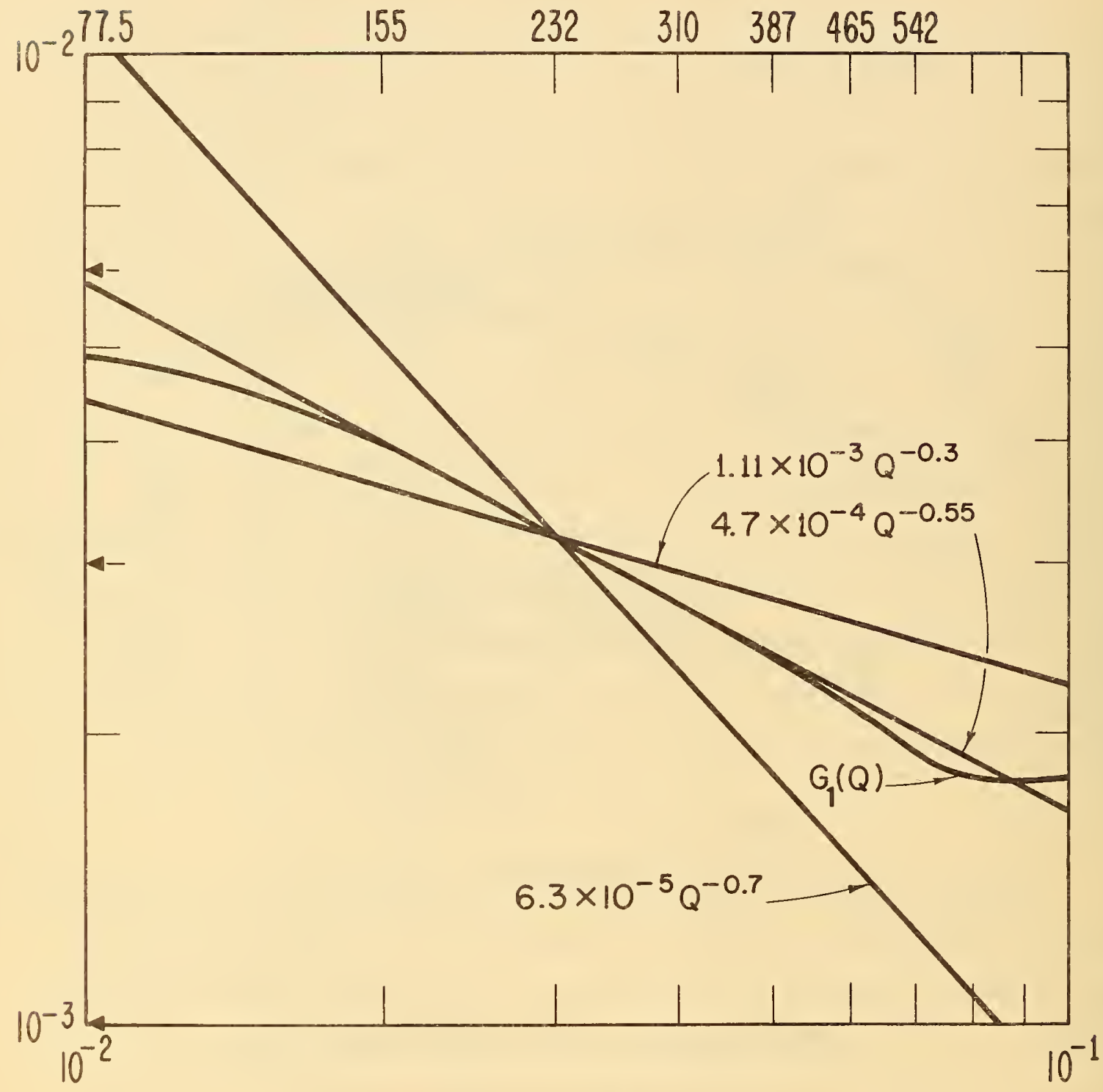

\section{ELECTRON ENERGY (Q), eV}

Figure 10. Energy dependence of the electron energy loss coefficient, G, within the range of electron temperatures encountered in the ionospheric D region. $G_{1}(Q)$ is taken as representing the best available laboratory determination, and the others are various analytical forms used in these studies. The arrows along the ordinate indicate the constant values of $G$ used. Electron temperature corresponds to the rms energy given. 


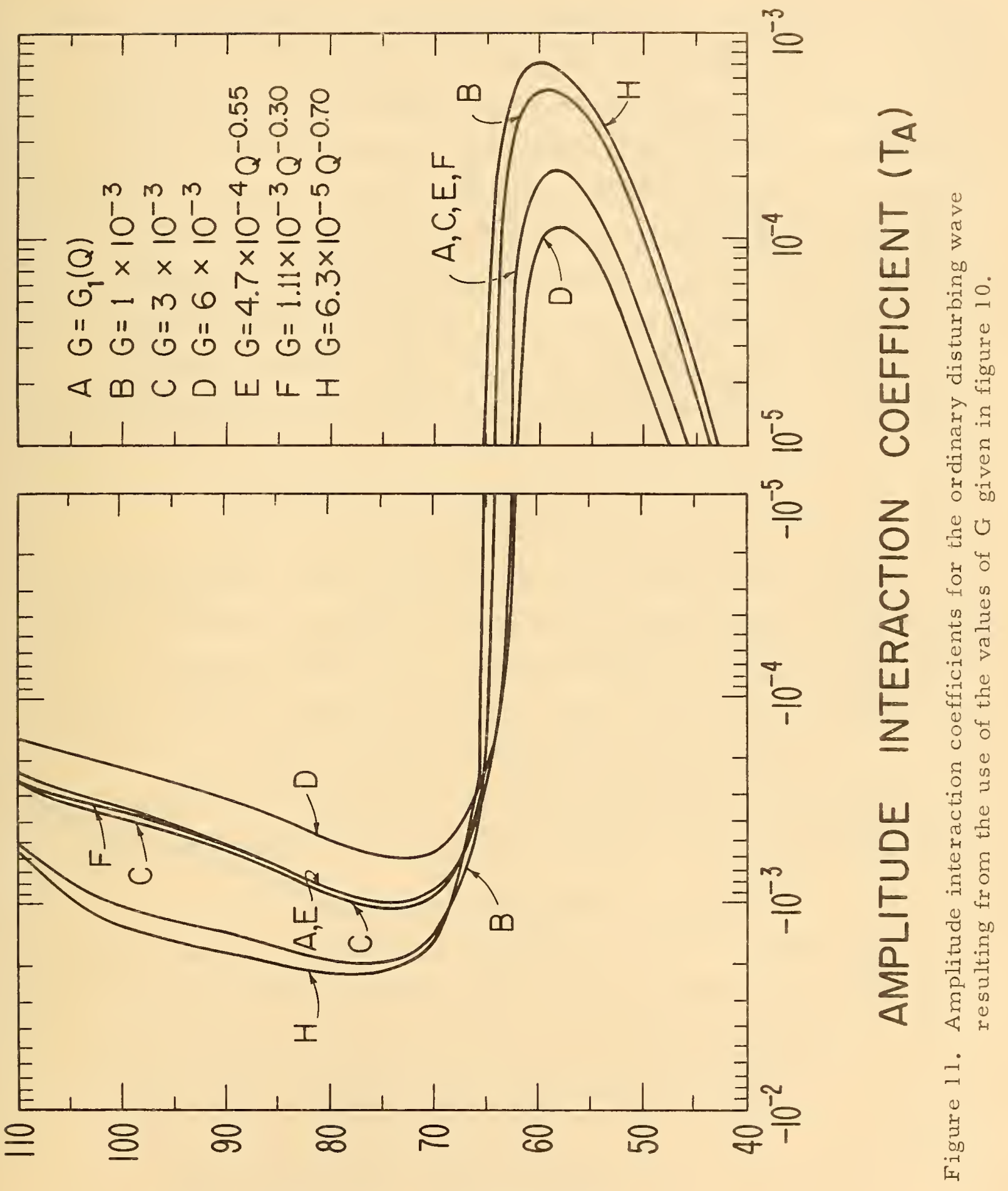

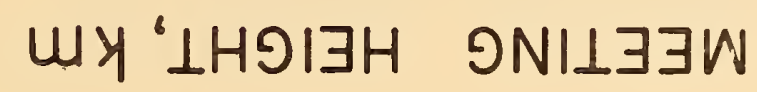


interaction profiles of other energy dependences. Calculations are also made for constant values of $G$, namely 1,3 , and $6 \times 10^{-3}$. The most profound effects on the interaction curves apparently result from dis placements of the $G(Q)$ curves rather than changes in slope, that is, changes in the coefficient rather than the exponent. Note particularly the relatively small difference between the $Q_{1}(Q)$ curve and the one using $G=3 \times 10^{-3}$. For the relatively small fractional changes in electron energy encountered here, the asymptotic approximation to $\mathrm{G}_{1}(\mathrm{Q})$ is satisfactory; however, for much higher disturbing powers (or lower frequencies) the tabular representation of the $G_{1}(Q)$ dependence would be required.

\subsection{Perturbations of the Electron Density Profile}

Figure 12 shows the effects on the interaction profile of changes in the electron density profile in the form of "bulges" inserted in $10 \mathrm{~km}$ intervals centered at the heights indicated. The bulges represent smooth increases in electron density of about $2 \frac{1}{2}$ times over the reference profile. One of these perturbed profiles is shown in figure 5 as curve $C^{\prime}$. The corresponding interaction profiles show maximum deviations of about $30 \%$, which of course depend somewhat on the height of the bulge.

\section{7 Disturbing Wave Self-distortion}

The degree of "self-distortion" of an originally rectangular disturbing pulse upon emergence from the absorbing region of the ionosphere is indicated in figure 13 for several variations in experimental parameters. The initial instant of the disturbing pulse is of course undisturbed, and the absorption increases with time, approaching the limit corresponding to conditions of a very long disturbing pulse. 

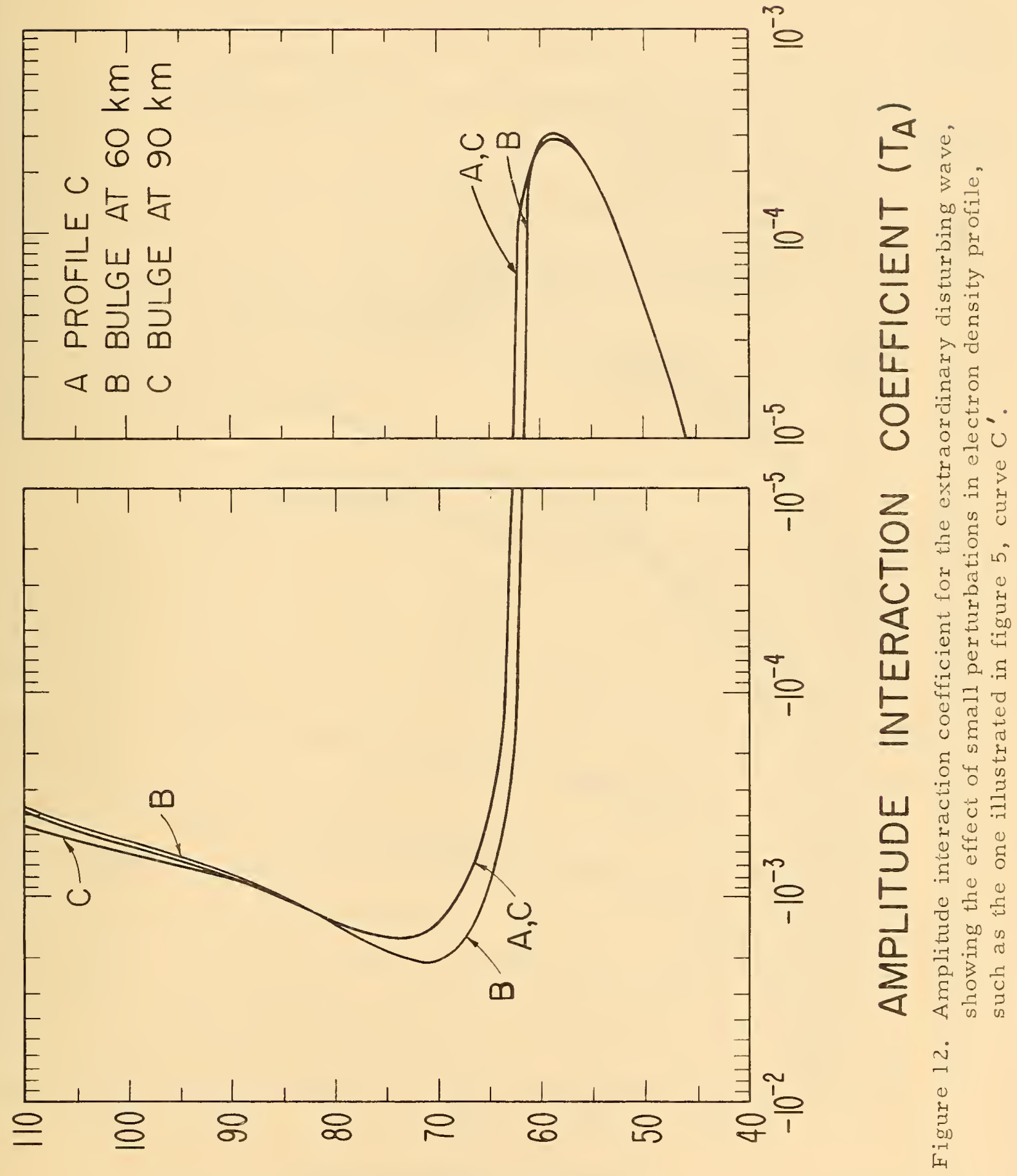

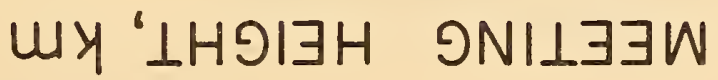




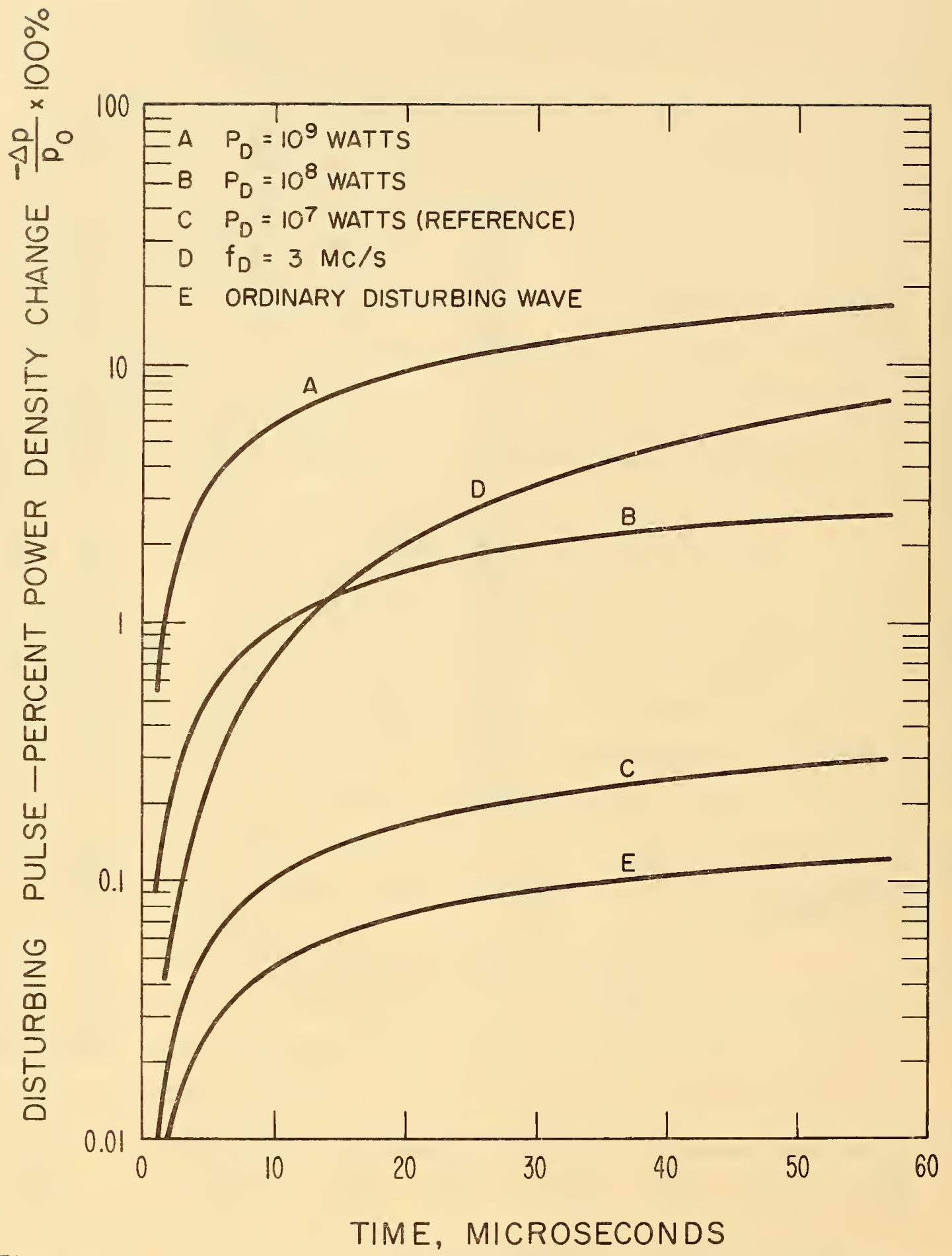

Figure 13. Self-distortion of the disturbing pulse shape as it emerges from the absorbing region of the ionosphere. At $t=0$ no self-distortion occurs, while near the end of the pulse the distortion approaches that of the limiting case of a very long disturbing pulse. 


\section{8 Use of the Generalized Theory}

Several calculations have been made using the general (arbitrary angle of propagation) refractive index expression of Sen and Wyller [1960]. When correction factors are applied to the values of collision frequency used in the Appleton-Hartree formulation, as indicated by Sen and Wyller, their expression leads to nearly the same interaction results as the complete Appleton-Hartree formulation within certain height ranges; however, no single correction factor brings the two into agreement over all heights. Figure 14 shows some results of these calculations.

\section{9 Gyro-interaction Effects}

Figure 9 has shown that conditions in the daytime $D$ region do not permit a sharp "resonance" in interaction as the disturbing frequency is varied about the gyrofrequency. However, figure 15, which represents nighttime conditions (profile A),shows a much sharper resonance which dominates the behavior of the interaction over a wide frequency range. At $f=f_{H}$ the gyrowave (extraordinary polarization) interaction is about $27 \mathrm{~dB}$ above that due to an ordinary wave at the same frequency. Evidently, absorption in the daytime D layer does not permit the gyrowave to reach heights where resonant effects can become important. Inspection of the absorption expression for the simple case of longitudinal propagation indicates that when $\nu$ is at least comparable in magnitude with $\omega$, the resonance in $\alpha$ as $\omega \rightarrow \omega_{H}$ will not be pronounced, i.e., it will have a low "Q":

$$
\alpha \sim \frac{N \nu}{\nu^{2}+\left(\omega-\omega_{H}\right)^{2}} .
$$




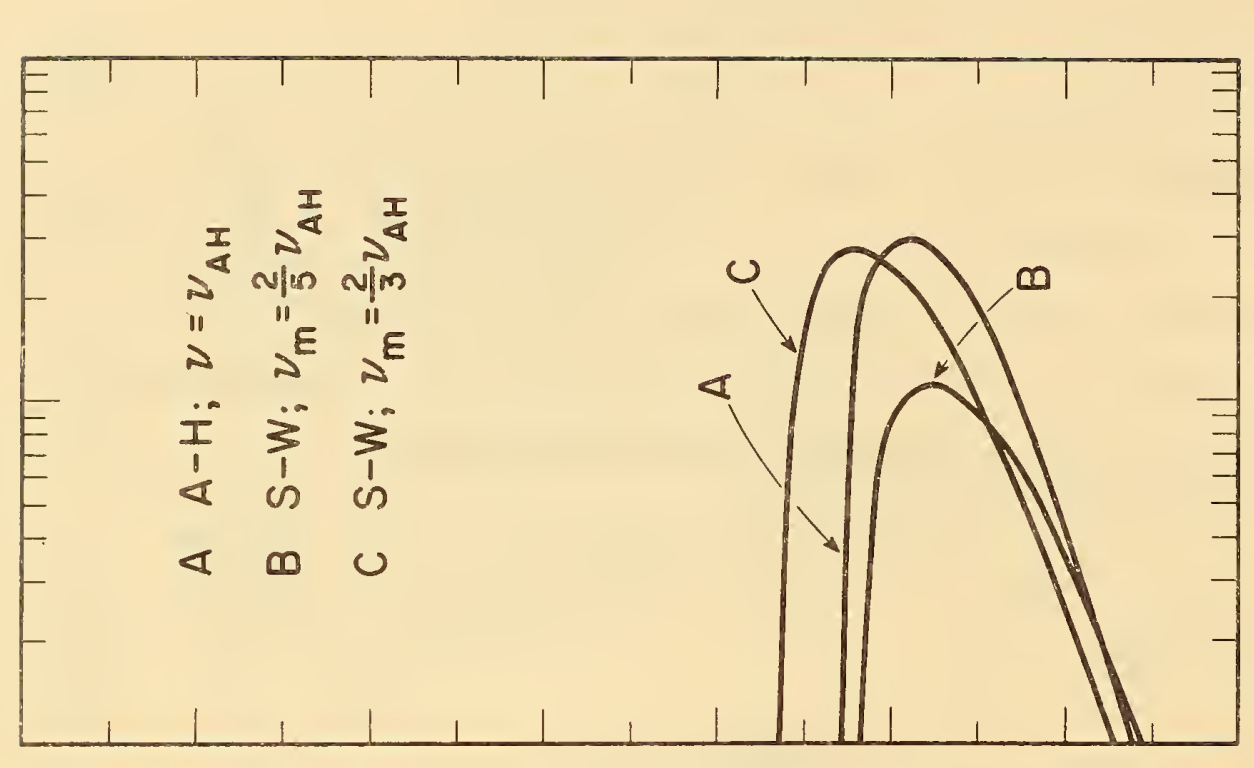

$\stackrel{10}{\circ}$

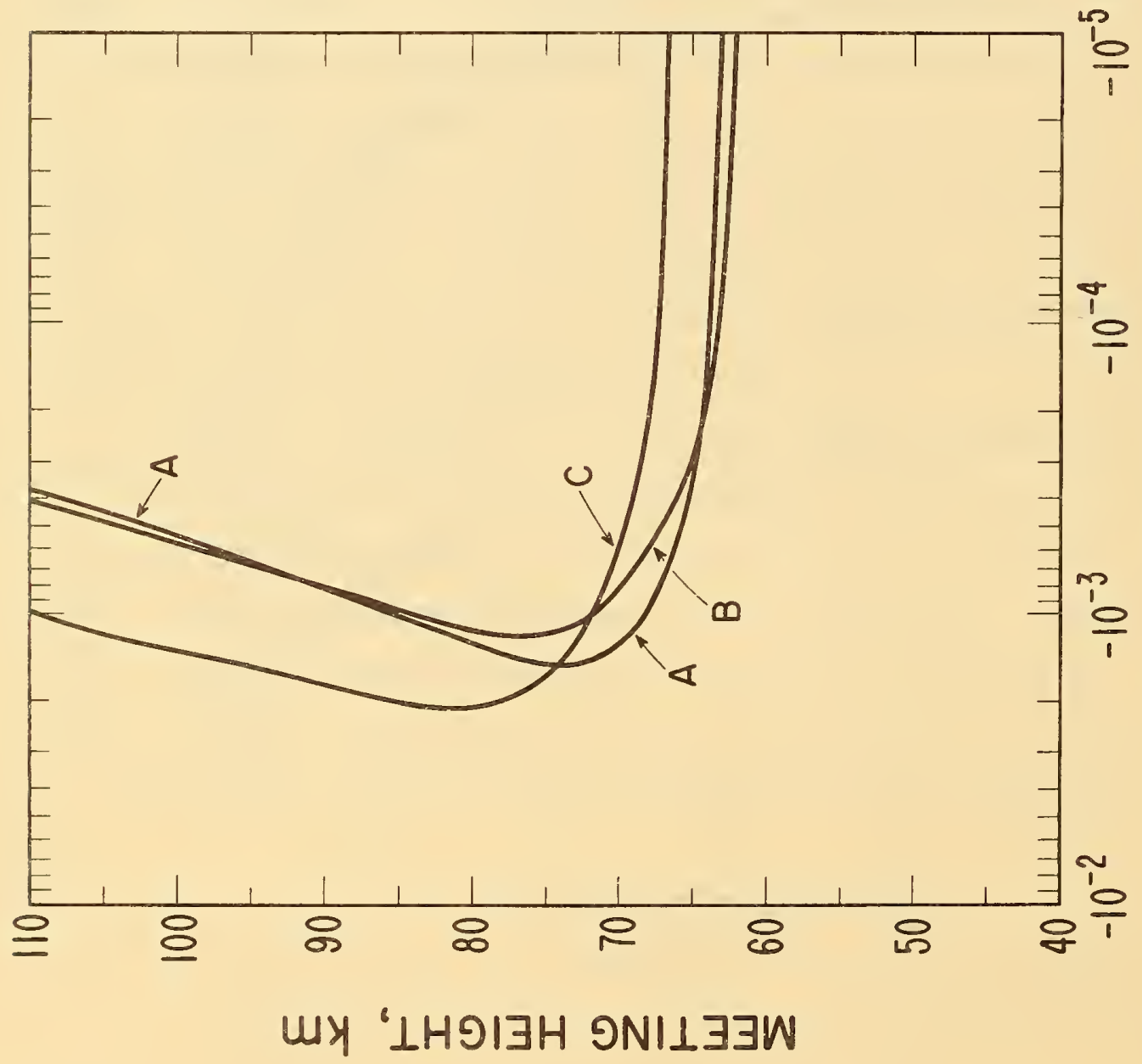

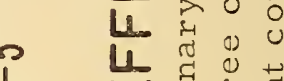

○ 山.

0 म 0 i⿱

त诘

¿ 10

$\geq 0 \begin{gathered}0 \\ 0\end{gathered}$

0 22 0

E

() क्ष 4

$\mathbb{1}_{4 \rightarrow}^{\circ}$

น

$\checkmark$ पा मू०

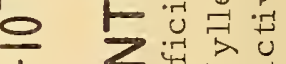

$\leq w_{3}$

11 14

U. 0 5 0

व

5.70 व

-

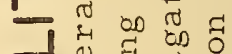

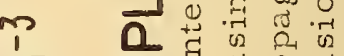

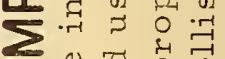

व 0 मे

उ

$\because=$

द्व $\begin{aligned} & 0 \\ & \text { ¿ }\end{aligned}$

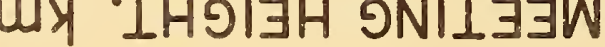




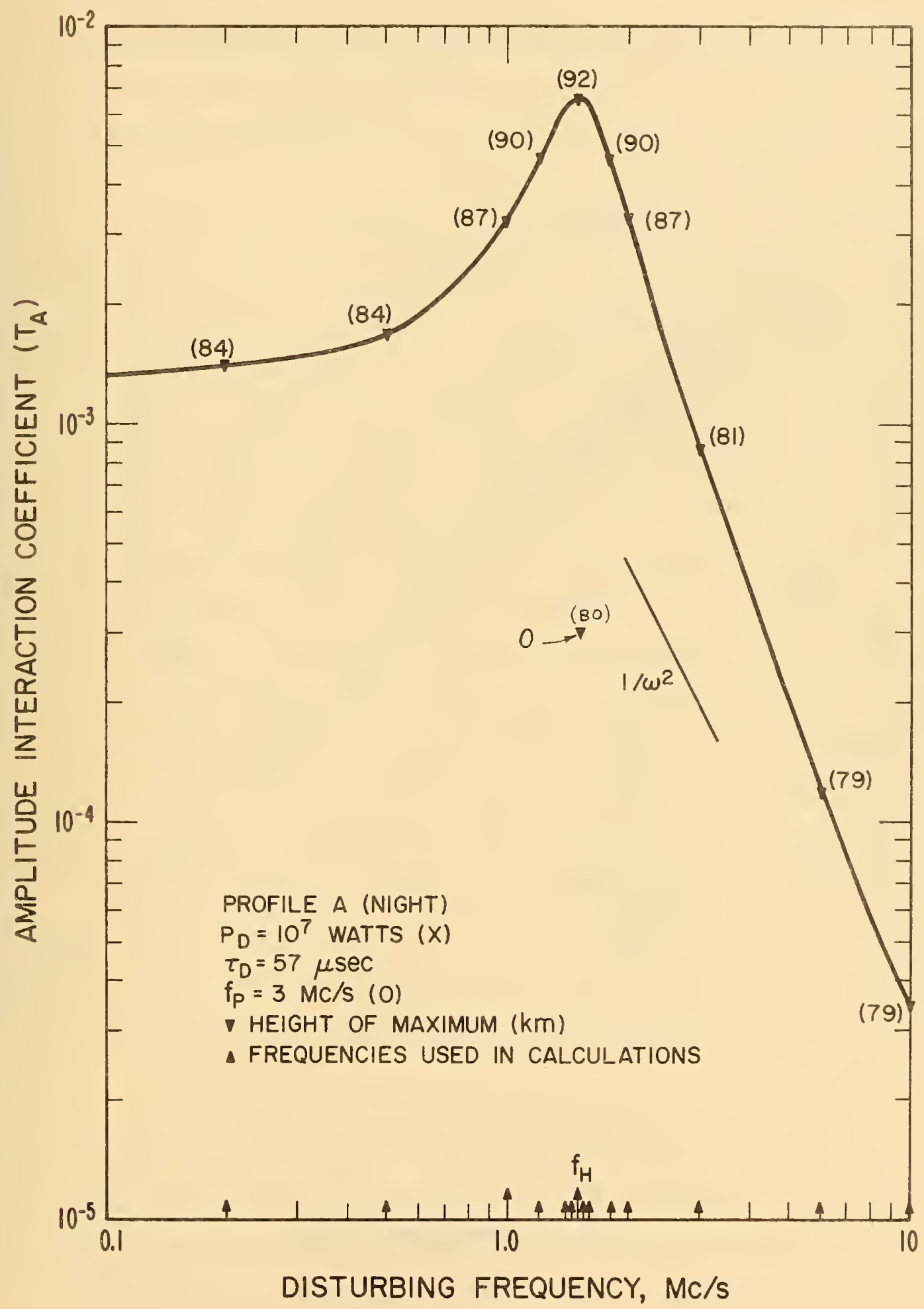

Figure 15. Maximum values of the amplitude interaction coefficient for nighttime conditions, for comparison with figure 9 (day). Curve is for the extraordinary disturbing polarization, and the value for the ordinary wave at $f_{H}$ is shown for comparison near the center of the figure. 
As $\nu$ decreases (at greater heights), the resonant effect becomes greater, until ray theory becomes inadequate due to effects which cause the relation between the electron velocity distribution and the wave phase velocity to become important.

An interesting manifestation of nighttime gyro-interaction effects is a large increase in electron energy and collision frequency. Figure 16 shows the maximum values of electron collision frequency attained during the passage of a 57 microsecond gyro-disturbing puls for various values of effective vertically radiated power. Note particularly the very high $\nu(h)$ gradients at the top of each disturbed region. For $10^{8}$ watts, the decrease is by almost a factor of 10 in a distance of one kilometer. Also, the disturbed region increases in height as power increases since the decrease in the absorption coefficient at lower heights allows penetration to greater heights before complete absorption occurs. This effect would not occur if selfinteraction were not accounted for. Daytime effects under similar conditions would hardly be noticeable on this figure.

In spite of the greatly increased interaction at the gyrofrequency, it is doubtful that useful ionosphere profiles can be deduced, since almost all of the interaction takes place in an interval only a few kilometers thick. On the other hand, this property can be used to advantage in the determination of $G, \nu$, and temperature within the interaction region.

\section{CONCLUSIONS AND LIMITATIONS}

It has been shown that a numerical simulation of ionospheric wave interaction can include several effects which are usually neglected in analytical calculations. This technique should prove useful in the evaluation of wave interaction experiments as well as in the development 


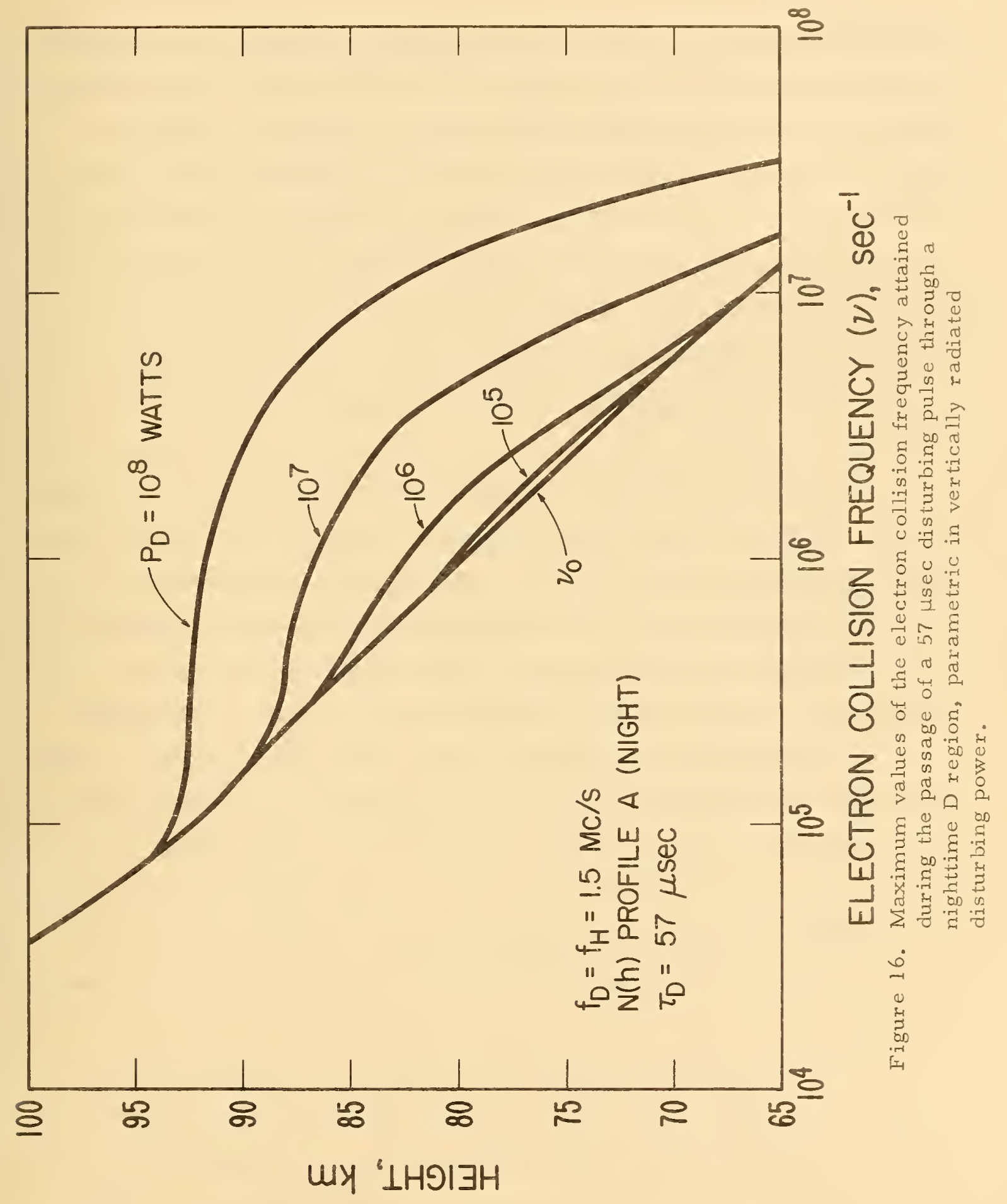


of refined theories of nonlinear ionospheric propagation. It is probable that the present state of the theory of wave interaction is not sufficiently sophisticated that the results presented here represent a degree of accuracy suitable for application to precise experimentation. The principal virtue of this method is that it is sufficiently flexible that new theoretical developments can be included without increasing the complexity of the basic interaction calculations. The need for such a technique has already become apparent since it does not appear possible to make interaction calculations using even today's most complete theories.

Perhaps the most limiting approximation which remains to be removed is the use of a propagation theory based on a Maxwellian form of the isotropic part of the electron energy distribution function (the Sen-Wyller treatment, for example). It is possible that for wave powers used in these experiments, the distribution can become appreciably non-Maxwellian under certain conditions. On the other hand, collision frequencies, particularly in the lower D region, may be sufficiently high that Maxwellian distributions are maintained even under very high-power conditions. Layzer and Menzel [1964] have discussed this problem for an amplitude-modulated disturbing wave, and Budden [1965] outlines an approach to the problem, but at this time no general solution in terms of wave propagation constants has yet appeared.

Another problem involves the "ray theory" approximations implicit in the present analysis; that is, that the medium exhibits only small space and time gradients with respect to the wave parameters. We have seen that at very low heights the heating time constant is measured in fractions of a microsecond, a time certainly not long compared to a wave period, particularly at lower frequencies. If 
sufficiently high power is used that collision frequency changes at low heights become appreciable, the ray approach may not be adequate.

Several conclusions regarding the optimization of experimental parameters are possible in view of the present calculations. Figure 9 indicates that the optimum disturbing frequency is in the vicinity of $10 \mathrm{Mc} / \mathrm{s}$, considering a trade off between available antenna gain, the $1 / \omega^{2}$ interaction variation, the applicability of ray theory, the possibility of $\mathrm{o} / \mathrm{x}$ separation, and the avoidance of interpretational complexities associated with the gyr ofrequency. Figure 7 shows that disturbing pulses shorter than about $50 \mu$ sec would not significantly improve height resolution, and that this is really a problem of probing pulse length. Much longer pulses are valuable in probing the higher regions and in exploring other nonlinear effects. Variations of disturbing and probing wave frequency and polarization can, under favorable circumstances, yield additional information, as seen from figure 8.

Figure 12 shows that the sensitivity to profile perturbations drops off at greater heights; consequently, this experiment appears to be most useful for obtaining profiles below about $85 \mathrm{~km}$. It should thus provide a useful extension to the capabilities of existing sweep-frequency vertical-incidence sounders.

The utility of cosmic-noise interaction [Benson, 1962] as a supplementary experimental technique should not be overlooked. It has the capability of providing information under conditions when the reflection technique is not useful, and eliminates the need for a smooth probing wave reflecting layer and the inherent restriction of probing frequencies; however, only amplitude interaction measurements are possible, and certain accuracy limitations are imposed by the random nature of the probing "signal". 
An obvious extension of the numerical simulation technique is the development of a numerical profile synthesis technique which yields $N(h)$ and $\nu(h)$ profiles given phase and amplitude interaction data. The two interaction coefficients are, in principle at least, capable of giving independent electron density and collision frequency profiles. It is easily seen, for example, that the ratio of $\delta \alpha_{p} / \delta \beta_{p}$ at each height depends to a good approximation on collision frequency at that height only. Since the pulse interaction experiment measures integrated absorption effects between the "base" of the ionosphere and each meeting height, any scheme for the synthesis of ionosphere profiles from interaction data must be either a trial-and-error profile-fitting process or an iterative procedure in which the difference between the interaction coefficients at two heights determines the contribution due to that height interval. Using a bootstrap approach, it is possible to obtain the $\delta \alpha_{p}$ and $\delta \beta_{p}$ contributions at each height from the interaction data. If the contributions due to a large number of height intervals can be obtained, $N(h)$ and $\nu(h)$ profiles can be calculated directly. The simulation program will provide the "data" for the development of this technique.

Acknowledgement is made of the considerable contributions by Dr. L. R. Megill to the initial development of this technique.

\section{REFERENCES}

Altshuler, S. (1963), Effects of inelastic collisions upon electrical conductivity and electron heating in the lower ionosphere, J. Geophys. Res. 68, No. 16, 4707-4716. Barrington, R, E., and $E$. Thrane (1962a), The determination of D-region electron densities from observations of cross modulations, J. Atmos. Terrest. Phys. 24, 31-42. 
Barrington, R. E., B. Landmark, and E. Thrane (1962b), Electron density profiles in the D-region deduced from observations of ionospheric cross modulation, Electron Density Profiles, NATO Conference Series 2 (MacMillan, New York).

Barrington, R. E., E. Thrane, and B. Bjelland (1963), Diurnal and seasonal variations in $\mathrm{D}$-region electron densities derived from observations of cross modulation, Can. J. Phys. 41, 271-285. Benson, R. F. (1962), Cross modulation of cosmic noise, J. Geophys. Res. 67, 2569-2572.

Benson, R. F. (1963), Cross modulation of cosmic noise: a technique for investigating the disturbed D-region, Scientific Report No. 1, Geophysical Institute, Univ. of Alaska.

Benson, R. F. (1964), A discussion of the theory of ionospheric crossmodulation, Radio Sci. J. Res. NBS 68D, No, 10, 1109-1122.

Budden, K. G. (1965), Effect of electron collisions on the formulas of magneto-ionic theory, Radio Sci. J. Res. NBS 69D, No. 1, $191-211$.

Fejer, J. A. (1955), The interaction of pulsed radio waves in the ionosphere, J. Atmos. Terrest. Phys. 7, 322-332.

Fejer, J. A. (1959), An investigation of the ionospheric D-region, J. Atmos. Terrest. Phys. 16, 291-306.

Ferraro, A. J., H. S. Lee and S. Weisbrod (1963), Phase interaction: a new tool for D-region studies, J. Geophys. Res. 68, $1169-1171$.

Huxley, L. G. H. (1953), Alternative developments of the theory of radio wave interaction, Proc. Roy. Soc, 218A, 520-536.

Knecht, R. W., and T. E. Van Zandt (1964), Chapter 6 in Space Physics, LeGalley and Rosen, Ed. (John Wiley and Sons, N. Y.). 
Layzer, D., and D. H. Menzel (1965), Ionospheric cross modulation: a microscopic theory, Radio Sci. J.Res. NBS 69D, No. 1, $59-68$.

Megill, L。 R。 (1965), Self-distortion of radio signals in the D-region, Radio Sci. J。 Res。NBS 69D, No. 3, 367-373.

Phelps, A. V., and J. L. Pack (1959), Electron collision frequencies in nitrogen and in the lower ionosphere, Phys. Rev. Letters $\underline{3}$, $340-342$.

Rumi, G. C., and C. G. Little (1958), Experiment Luxembourg, University of Alaska, Geophysical Institute Report 83.

Rumi, G. C., and R. F. Benson (1960), Experiment Luxembourg, University of Alaska, Geophysical Institute Report 111.

Rumi, G。C. (1961), Preliminary results of Experiment Luxembourg, J. Atmos. Terrest. Phys. 23, 101-105.

Rumi, G. C. (1962a), Experiment Luxembourg: cross modulation at high latitude, low height, Part I: Theoretical aspects, IRE Trans. Ant. Prop. AP-10, 594-600.

Rumi, G. C. (1962b), Experiment Luxembourg: cross modulation at high latitude, low height, Part II: Experimental aspects, IRE Trans. Ant. Prop. AP-10, 601-607.

Sen, $H_{0} K_{0}$, and A. A. Wyller (1960), On the generalization of the Appleton-Hartree magnetoionic formulas, J. Geophys. Res. 65, $3931-3950$.

Smith, R. A., V. A. Bailey, K. Landecker, A. J. Higgs, and F. H. Hibberd (1952), Resonance in gyro-interaction of radio waves, Nature 169, $911-913$.

Smith, R. A. (1964), Wave interaction research at the University of New England, NBS Tech. Note $211 \underline{4}, 39-44$.

Titheridge, J.E. (1962), The electron density in the lower ionosphere, J. Atmos. Terrest. Phys. 24, 269-282. 

U.S. DEPARTMENT OF COMMERCE

WASHINGTON, D.C. 20230

OFFICIAL BUSINESS
POSTAGE AND FEES PAID

U.S. DEPARTMENT OF COMMERCE 


BINDERY, INC,

EFEB.1973 


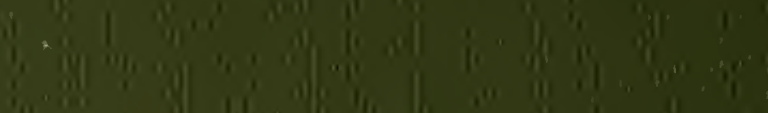

I0,

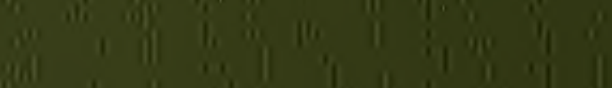

nin.

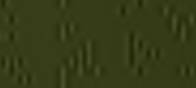

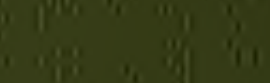

i n

6. 1.25

ล1 2.0

(II.

Tis.

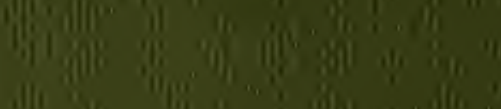

"

स' : :

' 